\title{
Inverse scattering by point-like scatterers in the Foldy regime
}

\author{
Durga Prasad Challa* Mourad Sini ${ }^{\dagger}$
}

July 13, 2012

\begin{abstract}
The scattering by point-like scatterers are described in the Born, Foldy and the intermediate regimes. We explain why the Foldy regime is, rigorously, a natural model for taking into account the multiple scattering. For each regime, we study the inverse problems for detecting these scatterers as well as the scattering strengths. In the first part, we do it for the acoustic case and in the second one we study the corresponding models for the linearized isotropic elastic case. In this last case, we show how any of the two body waves, namely the pressure waves $\mathrm{P}$ or the shear waves $\mathrm{S}$, is enough for solving the inverse problem. In the $3 \mathrm{D}$ case, it is shown that the shear-horizontal part $\mathrm{SH}$ or the shear vertical part SV of the shear waves S are also enough for the detection. Finally, we provide extensive numerical tests justifying our findings and discuss the question of resolution in terms of the distance between the scatterers, the used frequency and the scattering strengths. In addition, a comparison study between the three mentioned regimes is also provided.
\end{abstract}

Keywords: Acoustic scattering, Elastic scattering, Point-like scatterers, Multiple scattering, MUSIC algorithm.

\section{Introduction}

Scattering by point-like obstacles is well studied in many areas of applied sciences, as in quantum mechanics, acoustic and electromagnetic wave propagation, see [2], [12] and [25] for a review. A commonly used way of modeling the point-like obstacles is by considering the potentials (resp. the refraction indices) as highly concentrated coefficients on the different points so that they can be naturally considered as approximations of point sources, or Dirac impulses, see [12]. Following this point of view, one simplifies considerably the models. The price to pay is that, these coefficients being singular, the obtained models are no longer regular perturbations of known operators (as the Laplace operator in case of acoustic propagation). A way of representing the scattered wave, as a solution of these models, was first given by Foldy [14] who stated formally the fundamental equations of multiple scattering by finitely many point-like scatterers, see the system of equations (2.8)-(2.9). More details regarding this Foldy model and related works can be found in [12] and [25]. In parallel to this and motivated by applications in the field of quantum mechanics, several rigorous mathematical methods have been proposed to give sense to these singularly perturbed models and

*RICAM, Austrian Academy of Sciences, Altenbergerstrasse 69, A-4040, Linz, Austria. (Email: durga.challa@oeaw.ac.at) Supported by the Austrian Science Fund (FWF): P22341-N18.

${ }^{\dagger}$ RICAM, Austrian Academy of Sciences, Altenbergerstrasse 69, A-4040, Linz, Austria. (Email:mourad.sini@oeaw.ac.at) Partially supported by the Austrian Science Fund (FWF): P22341-N18. 
solve the scattering by N-particles. The general idea is to take this model as the one obtained by the limit, in the resolvent sense, of a sequence of operators generated by replacing the Dirac impulses by smoothed (or less singular) potentials. The arguments are based on the Weinstein-Aronszajn inversion formula or more generally on the Krein's inversion formulas for selfadjoint operators, see [2] for a comprehensive study of this issue. Due to the equivalence between the forms of the acoustic and the Schroedinger models, we can apply these techniques to the acoustic case as well. The result is that the represented solution using this obtained model is nothing but the Foldy model where the scattering coefficients (i.e. the scattering strengths) should be replaced by the renormalized ones, see Section 2.2 for more details on the validity of this Foldy model.

Following the ideas in [2], the corresponding model for the scattering by finitely many point-like scatterers for the Lamé system of equations is derived in [18]. Here the scattering is due to high concentrations of the densities on the scatterers which are, then, taken as Dirac impulses.

The purpose of our work here is to study an inverse problem type. Precisely, we are interested in reconstructing the point-like scatterers and the associated scattering strengths from the far fields corresponding to several incident plane waves. We use as models, (1) the Foldy one described above, taking into account the multiple scattering, (2) the Born approximation, neglecting the multiple scattering, and (3) the intermediate scattering models, taking into account a finite number of times the interactions between the scatterers. Due to the quasi-explicit form of the farfield patterns in all the models, we can justify and apply a MUSIC type algorithm for the reconstruction.

We start with the acoustic case where we describe the scattered fields in each of these models and then provide the inversion algorithm with several numerical tests discussing the resolution of the reconstruction depending on the number of scatterers, their distance, the used wavelength and the scattering strengths. Our focus will be on the target localization even though we provide also the corresponding formulas for recovering the scattering strengths. For this last issue, we cite the works $[11,13,23,24]$ for more insight and details on the actual implementations of those formulas.

As a second step, we describe the three models for the Lamé system and provide a justification of the MUSIC algorithm completing the work [17] where the Born approximation was used. In this Lamé system, we have two body waves, namely the pressure waves $\mathrm{P}$ or the shear waves $\mathrm{S}$. We show that anyone of these two waves is enough for solving the inverse problem. This observation was already made in [16] regarding the extended scatterers. In the 3D case, we show that, in addition, the shear-horizontal part SH-wave or the shear vertical part SV-wave of the shear waves are also enough for the detection. As for the acoustic case, we provide several numerical tests supporting these results and discuss the question of resolution. Remark that this question of resolution couldn't be discussed in [17] since the Born approximation is not appropriate for that. For both the acoustic and the elastic cases, a comparison study between the three mentioned models is provided. Finally, let us mention the following works concerning MUSIC type algorithms for detecting small inclusions using the near fields in elasticity $[4,5,6,19]$.

The rest of the paper is organized as follows. In Section 2, we study the acoustic scattering by point-like scatterers and then the corresponding inverse problems. In Section 3, we study the corresponding problems for the Lamé system. Finally, in Section 4, as an appendix, we give the detailed calculations for justifying the MUSIC algorithm for the Lamé system. 


\section{Acoustic scattering by point-like scatterers}

Let $U^{i}$ be a solution of the Helmholtz equation $\left(\Delta+\kappa^{2}\right) U^{i}=0$ in $\mathbb{R}^{d}, d=2,3$. Let also $U^{s}$ be the acoustic field scattered by a medium $D \subset \mathbb{R}^{d}$ due to the incident field $U^{i}$. The total field $U^{t}:=U^{i}+U^{s}$ satisfies the acoustic equation

$$
\left(\Delta+\kappa^{2} n\right) U^{t}=0 \text { in } \mathbb{R}^{d}, d=2,3
$$

and the scattered field $U^{s}$ satisfies the Sommerfield radiation condition :

$$
\lim _{|x| \rightarrow \infty}|x|^{\frac{d-1}{2}}\left(\frac{\partial U^{s}(x)}{\partial|x|}-i \kappa U^{s}(x)\right)=0 \text { uniformly in all directions } \hat{x}:=\frac{x}{|x|} \in \mathbb{S}^{d-1} .
$$

Here, $\kappa>0$ is the wavenumber and the scattering medium is modeled by the bounded and measurable index of refraction $n$ with $n=1$ outside of the bounded domain $D \subset \mathbb{R}^{d}$. We can rewrite the equation (2.1) as

$$
\left(\Delta+\kappa^{2}\right) U^{s}(y)=-\kappa^{2} q(y) U^{t}(y) \text { in } D .
$$

with $q:=n-1$ as the contrast. Recall that the fundamental solution of the Helmholtz equation is defined as $\Phi(x, y):=\frac{e^{i \kappa|x-y|}}{4 \pi|x-y|}$ in $\mathbb{R}^{3}$ and $\Phi(x, y):=\frac{i}{4} \mathbf{H}_{0}^{1}(\kappa|x-y|)$ in $\mathbb{R}^{2}$, where $\mathbf{H}_{0}^{1}$ is the Hankel function of the first kind and order zero. The scattering problem (2.1)-(2.2) is well posed, see [7,8] for instance.

Multiplying the equation (2.3) by the fundamental solution and applying integration by parts, we obtain the following Lippmann-Schwinger equation

$$
U^{t}(x)=U^{i}(x)+\kappa^{2} \int_{D} q(y) \Phi(x, y) U^{t}(y) d y .
$$

To describe the scattering by $M$ point-like scatterers $y_{1}, y_{2}, \ldots, y_{M}, M \in \mathbb{N}$, we follow De Vries-van CoevordenLagendijk and take $q$ as $q(y):=\frac{1}{\kappa^{2}} \sum_{m=1}^{M} g_{m} \delta\left(y-y_{m}\right), g_{m} \in \mathbb{R}, m=1, \ldots, M$, see $[25,12]$. Here, $\delta$ is the Dirac measure and $g_{m}$ 's are the scattering strengths of the point-like scatterers $y_{m}$ 's. Now, we can write equation (2.4) formally as

$$
U^{t}(x)=U^{i}(x)+\sum_{m=1}^{M} g_{m} \Phi\left(x, y_{m}\right) U^{t}\left(y_{m}\right), x \neq y_{m}, m=1,2, \ldots, M .
$$

To use the formula $(2.5)$, we need to know $U^{t}\left(y_{m}\right)$. However, we cannot calculate $U^{t}\left(y_{m}\right), m=$ $1,2, \ldots, M$ : we cannot evaluate $(2.5)$ at $x=y_{m}$ due to the singularity of $\Phi\left(x, y_{m}\right)$ at $x=y_{m}$. Therefore, few approximations were introduced, see [25] for more information concerning this issue. In the next sections, we discuss the Born approximation, the Foldy method and then the intermediate levels of scattering.

\subsection{Approximation methods}

\subsubsection{Born approximation}

In the Born approximation, the total field $U^{t}\left(y_{m}\right)$, at the point-like scatterer $y_{m}$, is replaced by the incident field $U^{i}\left(y_{m}\right)$ in equation (2.5). It means that the Born approximation neglects the effect of multiple scattering and deals with weak scattering. We obtain the following representation of the total field

$$
U^{t}(x)=U^{i}(x)+\sum_{m=1}^{M} g_{m} \Phi\left(x, y_{m}\right) U^{i}\left(y_{m}\right)
$$


and then the scattered field is given by

$$
U^{s}(x)=\sum_{m=1}^{M} g_{m} \Phi\left(x, y_{m}\right) U^{i}\left(y_{m}\right)
$$

Using plane waves, $U^{i}(x, \theta)=e^{i \kappa x \cdot \theta}$ with direction of incidence $\theta \in \mathbb{S}^{d-1}$, and knowing the asymptotic expansion of $\Phi(x, y)$ as $|x| \rightarrow \infty$, we obtain the far field pattern related to the Born approximation as

$$
U^{\infty}(\hat{x}, \theta)=\sum_{m=1}^{M} g_{m} e^{i \kappa y_{m} \cdot(\theta-\hat{x})}, \hat{x}, \theta \in \mathbb{S}^{d-1} .
$$

\subsubsection{Foldy's method}

In contrast to the Born approximation, and following the method of Foldy, used also for the scattering by many small obstacles, see $[14,25]$, we replace the equation $(2.5)$ by

$$
U^{t}(x)=U^{i}(x)+\sum_{m=1}^{M} g_{j} \Phi\left(x, y_{m}\right) U_{m}\left(y_{m}\right)
$$

where the terms $U_{m}\left(y_{m}\right)$ 's are calculated from the Foldy algebraic system given by

$$
U_{m}\left(y_{m}\right)=U^{i}\left(y_{m}\right)+\sum_{\substack{j=1 \\ j \neq m}}^{M} g_{m} \Phi\left(y_{m}, y_{j}\right) U_{j}\left(y_{j}\right), \forall m=1, \ldots, M .
$$

In [14], (2.8)-(2.9) are called the fundamental equations of multiple scattering. From the system (2.9), we see how the Foldy method takes into account the multiple scattering effect between the scatterers.

From (2.8)-(2.9), we obtain the scattered field $U^{s}(x)$ as

$$
U^{s}(x)=\sum_{m=1}^{M} g_{m} \Phi\left(x, y_{m}\right) U_{m}\left(y_{m}\right) .
$$

In particular, for plane incident waves $U^{i}(x, \theta)=e^{i \kappa x \cdot \theta}$ we obtain the far field pattern related to Foldy method as

$$
U^{\infty}(\hat{x}, \theta)=\sum_{m=1}^{M} g_{m} e^{-i \kappa \hat{x} \cdot y_{m}} U_{m}\left(y_{m}\right), \hat{x}, \theta \in \mathbb{S}^{d-1} .
$$

\subsubsection{Intermediate levels of scattering}

Here, we give a common platform which deals with intermediate levels of scattering between the Born and Foldy models. For any non negative integer $k$, let $U_{k}^{t}$ and $U_{k}^{s}$ denote the total and the scattered fields respectively in the $k^{t h}$ level scattering. We set $U_{m, 0}=U^{i}, \forall m=1, \ldots, M$, to be the incident wave. Then the total field in the $k^{t h}$ level scattering is calculated by

$$
U_{k}^{t}(x)=U^{i}(x)+\sum_{m=1}^{M} g_{m} \Phi\left(x, y_{m}\right) U_{m, k}\left(y_{m}\right),
$$


where the terms $U_{m, k}\left(y_{m}\right)$ 's, indicating the exciting fields, are defined recursively by

$$
U_{m, k+1}\left(y_{m}\right):=U^{i}\left(y_{m}\right)+\sum_{\substack{j=1 \\ j \neq m}}^{M} g_{j} \Phi\left(y_{m}, y_{j}\right) U_{j, k}\left(y_{j}\right) \text { for } m=1, \ldots, M .
$$

From (2.12)-(2.13), the scattered field $U_{k}^{s}(x)$ in the $k^{t h}$ level scattering is given by

$$
U_{k}^{s}(x)=\sum_{m=1}^{M} g_{m} \Phi\left(x, y_{m}\right) U_{m, k}\left(y_{m}\right)
$$

From the above equations, we observe that $k=0 \& \infty$ deal with the Born approximation and the Foldy model respectively. The system (2.13) is nothing but the $k+1^{t h}$ iteration of the Foldy algebraic system (2.9). In particular, for plane incident waves $U^{i}(x, \theta)=e^{i \kappa x \cdot \theta}$ we obtain the far field pattern related to the $k^{\text {th }}$ level scattering as

$$
U_{k}^{\infty}(\hat{x}, \theta)=\sum_{m=1}^{M} g_{m} e^{-i \kappa \hat{x} \cdot y_{m}} U_{m, k}\left(y_{m}\right) .
$$

For each of these models, we study the following inverse problem.

Inverse Problem : Given the far field pattern $U^{\infty}(\hat{x}, \theta)$ for several incident and observation angles $\theta$ and $\hat{x}$, locate the point-like scatterers $y_{1}, y_{2}, \ldots, y_{M}$ and reconstruct the scattering strengths $g_{1}, g_{2}, \ldots, g_{M}$.

\subsection{The validity of the Born, Foldy and intermediate models}

- Foldy's model. First, we observe that the Foldy algebraic system (2.9) is obtained from (2.5) by taking $x$ tend to $y_{m}, m=1, \ldots, M$ and deleting the singular term in the sum. There are several ways to justify and give sense to this step. The first one is related to the regularization of the model (2.1), or (2.3). We mention the reference ([2], chapter II) where this is studied in the frame work of interactions of point-like particles in quantum mechanics. We highlight the main idea behind this method. Replacing the scattering coefficients $g_{m}$ by the parameter dependent coefficients $g_{m}(\varsigma):=\left(g_{m}^{-1}+\frac{\varsigma}{2 \pi^{2}}\right)^{-1}, \varsigma \in \mathbb{R}_{+}$, and the Fourier transform of the delta distribution by its truncated part, up to $\varsigma$, they obtain a parameter family of self-adjoint operators, with $\varsigma$ as a parameter, in the Fourier variable. These operators are finite-rank perturbations of the multiplication operator (which is the Fourier transform of the Laplacian). Based on the Weinstein-Aronszajn formula, they show that the resolvent of this family of operators converges, as $\varsigma \rightarrow \infty$, to the resolvent of a closed and self-adjoint operator which they define as the Fourier transform of the operator modeling the scattering by finitely many point-like obstacles. As a result, the scattering fields, computed via the resolvent of this operator, is represented by nothing but $(2.10)$ where $g_{m}$ is replaced by $\left(g_{m}^{-1}-\frac{i \kappa}{4 \pi}\right)^{-1}$, i.e. exactly the Foldy representation. We can deduce then that the Foldy model is a natural model to describe the multiple interactions of point-like obstacles. Let us also mention that approximating models of the form (2.3) replacing $q$ by less singular potentials than the delta-type potentials, i.e. the compactly supported Rollnik potentials, are provided in [2, chapter II]. It is proved that the corresponding family of self-adjoint operators converge in the norm resolvent sense to the operator modeling the scattering by finitely many pointlike obstacles described above. The scattering strengths $g_{m}$ 's are related to the limits of those Rollnik potentials. 
A second way to justify the Foldy model is demonstrated in details in [15], see also [2], where the Krein formula of the resolvent of the extensions of self-adjoint operators is used, instead of the WeinsteinAronszajn determinant formula. This provides a more general representation of the scattered field due to point-like scatterers where the Foldy model is a particular one.

- Born's approximation. Assuming that the points scatterers are far away from each other, i.e. $\mid y_{i}-$ $y_{j} \mid>>1, i \neq j$, then a good approximate solution of the linear system (2.9) is indeed the vector $\left(U^{i}\left(y_{j}\right)\right)_{j=1, \ldots, M}$. This implies that the Foldy model reduces to the Born model.

- Intermediate levels of scattering. Let $k=1$, then

$$
U_{m, 1}\left(y_{m}\right)=U^{i}\left(y_{m}\right)+\sum_{j \neq m} g_{j} \Phi\left(y_{m}, y_{j}\right) U^{i}\left(y_{j}\right)
$$

which means that the total field on the point scatterer $y_{m}$ is given by the incident wave $U^{i}\left(y_{m}\right)$ plus the scattered field by each of the other scatterers, $y_{j} j \neq m$, taken separately. This model takes into account one time interaction between the scatterers.

Let $k=2$, then

$$
U_{m, 2}\left(y_{m}\right)=U^{i}\left(y_{m}\right)+\sum_{j \neq m} g_{j} \Phi\left(y_{m}, y_{j}\right) U_{m, 1}
$$

which we can write, using (2.16), as

$$
U_{m, 2}\left(y_{m}\right)=U^{i}\left(y_{m}\right)+\sum_{j \neq m} g_{j} \Phi\left(y_{m}, y_{j}\right) U^{j}\left(y_{m}\right)+\sum_{j \neq m} g_{j} \Phi\left(y_{m}, y_{j}\right)\left[\sum_{j \neq m} g_{j} \Phi\left(y_{m}, y_{j}\right) U^{j}\left(y_{m}\right)\right] .
$$

This means that the total field on the point scatterer $y_{m}$ is given by the incident field $U^{i}\left(y_{m}\right)$ plus the scattered field, due the incident field $U^{i}$, by the each of the other scatterers, $y_{j} j \neq m$, taken separately and plus the scattered field of each scatterer $y_{j}, j \neq m$, due to the incident field given by the scattered wave by the other scatterers $y_{s}, s \neq j$. This model takes into account the two-level interaction between the scatterers.

Iterating this process, we can see how the $k^{\text {th }}$ level of scattering takes into account the $k$-level interactions between the scatterers.

\subsection{The inverse problems for the Born and Foldy models}

\subsubsection{Localisation of $y_{m}$ 's via the MUSIC algorithm}

The MUSIC algorithm is a method to determine the locations $y_{m}, m=1,2, \ldots, M$, of the scatterers from the measured far field pattern $U^{\infty}(\hat{x}, \theta)$ for a finite set of incidence and observation directions, i.e. $\hat{x}, \theta \in$ $\left\{\theta_{j}, j=1, \ldots, N\right\} \subset \mathbb{S}^{d-1}$. We refer the reader to the monographs [3] and [20] for more information about this algorithm. We follow the way presented in [20]. We assume that the number of scatterers is not larger than the number of incident and observation directions, i.e. $N \geq M$. We define the response matrix $F \in \mathbb{C}^{N \times N}$ by

$$
F_{j l}:=U^{\infty}\left(\theta_{j}, \theta_{l}\right) .
$$

In order to determine the locations $y_{m}$, we consider a grid of sampling points $z \in \mathbb{R}^{d}$ in a region containing the scatterers $y_{1}, y_{2}, \ldots, y_{M}$. For each point $z$, we define the vector $\phi_{z} \in \mathbb{C}^{N}$ by

$$
\phi_{z}:=\left(e^{-i \kappa \theta_{1} \cdot z}, e^{-i \kappa \theta_{2} \cdot z}, \ldots, e^{-i \kappa \theta_{N} \cdot z}\right)^{T} .
$$


MUSIC characterisation of the scatterers: The MUSIC algorithm is based on the property that $\phi_{z}$ is in the range $\mathcal{R}(F)$ of $F$ if and only if $z$ is at one of locations of the scatterers. Precisely, let $\mathcal{P}$ be the projection onto the null space $\mathcal{N}\left(F^{*}\right)=\mathcal{R}(F)^{\perp}$ of the adjoint matrix $F^{*}$ of $F$, then

$$
z \in\left\{y_{1}, y_{2}, \ldots, y_{M}\right\} \Longleftrightarrow \mathcal{P} \phi_{z}=0 .
$$

This property can be proved based on the factorization $F=H^{*} T H$ of $F \in \mathbb{C}^{N \times N}$, where the matrix $T \in \mathbb{C}^{M \times M}$ is invertible and $H \in \mathbb{C}^{M \times N}$ defined in terms of the vectors $\phi_{y_{1}}, \phi_{y_{2}}, \ldots, \phi_{y_{M}}$ has a maximal rank.

For the Born approximation, this factorization is clear from (2.7) with $T:=\operatorname{Diag}\left(g_{1}, g_{2}, \ldots, g_{M}\right) \in \mathbb{C}^{M \times M}$, the diagonal matrix with diagonal entries as $g_{m}$ 's, and $H \in \mathbb{C}^{M \times N}$, defined by $H_{p q}:=e^{i \kappa \theta_{q} \cdot y_{p}}, 1 \leq p \leq$ $M, 1 \leq q \leq N$. The maximal rank property of $H$ is justified in [20, chapter 4] for instance. ${ }^{1}$

In the case of Foldy, we can write the Foldy algebraic system (2.9) in a compact form as

$$
A \mathbf{U}_{I}=\mathbf{U}^{I}
$$

where the matrix $A \in \mathbb{C}^{M \times M}$ and the vectors $\mathbf{U}_{I}, \mathbf{U}^{I} \in \mathbb{C}^{M \times 1}$ are given by

$$
\begin{gathered}
A:=\left(\begin{array}{ccccc}
1 & -g_{2} \Phi\left(y_{1}, y_{2}\right) & -g_{3} \Phi\left(y_{1}, y_{3}\right) & \ldots & -g_{M} \Phi\left(y_{1}, y_{M}\right) \\
-g_{1} \Phi\left(y_{2}, y_{1}\right) & 1 & -g_{3} \Phi\left(y_{2}, y_{3}\right) & \ldots & -g_{M} \Phi\left(y_{2}, y_{M}\right) \\
\ldots & \ldots & \ldots & \ldots & \ldots \\
-g_{1} \Phi\left(y_{M}, y_{1}\right) & -g_{2} \Phi\left(y_{M}, y_{2}\right) & -g_{3} \Phi\left(y_{M}, y_{3}\right) & \ldots & 1
\end{array}\right), \\
\mathbf{U}_{I}:=\left[U_{1}\left(y_{1}\right), U_{2}\left(y_{2}\right), \ldots, U_{M}\left(y_{M}\right)\right]^{T} \text { and } \mathbf{U}^{I}:=\left[U^{i}\left(y_{1}\right), U^{i}\left(y_{2}\right), \ldots, U^{M}\left(y_{M}\right)\right]^{T} .
\end{gathered}
$$

We suppose that $A$ is non-singular and denote its inverse by $B:=\left(b_{i j}\right) \in \mathbb{C}^{M \times M}$. From (2.19), we obtain

$$
\mathbf{U}_{I}=B \mathbf{U}^{I}
$$

Then using (2.11), the response matrix $F \in \mathbb{C}^{N \times N}$ can be factorized as

$$
F=H^{*} T H
$$

where, in this case, $T:=\operatorname{Diag}\left(g_{1}, g_{2}, \ldots, g_{M}\right) B$ while $H$ is the same matrix we introduced before.

In the case of Born approximation, it is clear that $T$ is invertible. Let us deal with the Foldy case. We observe that the matrix $A \in \mathbb{C}^{M \times M}$ can be factorized as $A=\mathcal{A g}$ with $\mathcal{A} \in \mathbb{C}^{M \times M}$ defined by

$$
\mathcal{A}:=\left(\begin{array}{ccccc}
\frac{1}{g_{1}} & -\Phi\left(y_{1}, y_{2}\right) & -\Phi\left(y_{1}, y_{3}\right) & \ldots & -\Phi\left(y_{1}, y_{M}\right) \\
-\Phi\left(y_{2}, y_{1}\right) & \frac{1}{g_{2}} & -\Phi\left(y_{2}, y_{3}\right) & \ldots & -\Phi\left(y_{2}, y_{M}\right) \\
\ldots & \ldots & \ldots & \ldots & \ldots \\
-\Phi\left(y_{M}, y_{1}\right) & -\Phi\left(y_{M}, y_{2}\right) & -\Phi\left(y_{M}, y_{3}\right) & \ldots & \frac{1}{g_{M}}
\end{array}\right) \text { and } g:=\operatorname{Diag}\left(g_{1}, g_{2}, \ldots, g_{M}\right)
$$

Then $T=\mathbf{g} B=\mathbf{g} A^{-1}=\mathbf{g}(\mathcal{A g})^{-1}=\mathcal{A}^{-1}$. Hence, it is enough to consider the invertibility of $\mathcal{A}$.

\footnotetext{
${ }^{1}$ We show the idea and the details of the proof in the appendix in framework of the inverse elastic scattering.
} 


\subsubsection{Invertibility of the matrix $\mathcal{A}$}

We discuss here the conditions under which the matrix $\mathcal{A} \in \mathbb{C}^{M \times M}$ is invertible.

Case 1 (Diagonally dominant condition):

As the matrix $\mathcal{A}$ is symmetric, row wise and the column wise diagonally dominant conditions match. The diagonally dominant condition for $\mathcal{A}$ is,

$$
\sum_{\substack{i=1 \\ i \neq j}}^{M}\left|\Phi\left(y_{i}, y_{j}\right)\right|<\frac{1}{\left|g_{j}\right|}, \forall j=1,2, \ldots, M
$$

We have, $\Phi(x, y)=\Phi(y, x):=\frac{e^{i k|x-y|}}{4 \pi|x-y|}$ in $\mathbb{R}^{3}$ and $\Phi(x, y)=\Phi(y, x):=\frac{i}{4} \mathbf{H}_{0}^{1}(\kappa|x-y|)$ in $\mathbb{R}^{2}$. In $\mathbb{R}^{3},(2.21)$ can be written as

$$
\sum_{\substack{i=1 \\ i \neq j}}^{M}\left|\Phi\left(y_{i}, y_{j}\right)\right|<\frac{1}{\left|g_{j}\right|} \Longleftrightarrow \sum_{\substack{i=1 \\ i \neq j}}^{M} \frac{1}{\left|y_{i}-y_{j}\right|}<\frac{4 \pi}{\left|g_{j}\right|}, \forall j=1,2, \ldots, M .
$$

The relation (2.22) tells us that if the scattering strength of every point-like scatterer $y_{j}, j=1,2, \ldots, M$, is less than $4 \pi$ over the sum of the reciprocals of the distance of the scatterer $y_{j}, j=1,2, \ldots, M$, from the other scatterers, then $\mathcal{A}$ is invertible.

In particular, the condition that the left part of $(2.21)$ is much smaller $(\ll)$ than its right part for every $j$, (i.e. when the scatterers are relatively far away from each other compared to the scattering strengths) leads to weak scattering, for example Born approximation.

Case 2 (Non-diagonally dominant condition):

The necessary and sufficient condition for the invertibility of $\mathcal{A}$ in the case of two scatterers is $\Phi^{2}\left(y_{1}, y_{2}\right) \neq$ $\frac{1}{g_{1} g_{2}}$. Fixing the wavenumber $\kappa$ and the scattering strengths $g_{1}$ and $g_{2}$, this condition holds almost every time except for the distributions of the scatterers satisfying $\left|y_{1}-y_{2}\right|^{2}=\frac{1}{16 \pi^{2}}\left|g_{1}\right|\left|g_{2}\right|$ in 3D case for instance. These exceptions where $\mathcal{A}$ is singular are called resonances, see [2, Chapter II.1]. This observations can be generalized to the case of finitely many point-like scatterers as follows. Fix the wavenumber $\kappa$ and the scattering strengths $g_{1}, g_{2}, \ldots, g_{M}$ and look at $\operatorname{det} \mathcal{A}$ as a function of the $\frac{M(M-1)}{2}$ - real variables $\xi_{i j}:=\left|y_{i}-y_{j}\right|$ for $i, j=1, \ldots, M$ with $i<j$ and set $\xi:=\left(\xi_{1,2}, \xi_{1,3}, \ldots, \xi_{1, M}, \xi_{2,3}, \ldots, \xi_{M-1, M}\right)$. Then due to the explicit form of $\mathcal{A}$, we see that $\xi \rightarrow \operatorname{det} \mathcal{A}(\xi)$ is a real analytic function in $\mathbb{R}_{+}^{\frac{M(M-1)}{2}}$. Hence, it has locally a finite number of zeros. These zeros are related to the possible distributions of the scatterers for which Foldy's method does not apply.

\subsubsection{Recovering the scattering strengths $g_{m}$ 's}

Once we locate the scatterers from the given far field patterns using the MUSIC algorithm, we can recover the scattering strengths from the factorization of $F \in \mathbb{C}^{N \times N}$. Indeed, from Theorem 4.1 of [20], we know that the matrix $H$ has maximal rank, see also the appendix in Section 4. So, the matrix $H H^{*} \in \mathbb{C}^{M \times M}$ is invertible. Let us denote its inverse by $I_{H}$. Once we locate the scatterers $y_{1}, y_{2}, \ldots, y_{M}$ by using the MUSIC algorithm for the given far field patterns, we can recover $I_{H}$ and hence the matrix $T \in C^{M \times M}$ given by $T=I_{H} H F H^{*} I_{H}$, where $I_{H} H$ is the pseudo inverse of $H^{*}$. As we know the structure of $T \in \mathbb{C}^{M \times M}$ in both Born $(T=\mathbf{g})$ and Foldy $\left(T=\mathcal{A}^{-1}\right)$ approximations, we can recover the scattering strengths $g_{1}, \ldots, g_{M}$ from the diagonal entries of $T$ or of $T^{-1}$ in Born approximation and Foldy model respectively. 


\subsubsection{Numerical results and discussions}

In this section, we illustrate the performance of the MUSIC algorithm for this acoustic case and present results for locating the scatterers using Foldy method. We also present the results for comparing weak (Born) and multiple (Foldy) scatterings.

For the convenience of visualization, we only show the results for two dimensional problems. However, we should mention that the algorithm in two and three dimensional spaces are the same. Denote by $N_{d}$ the number of incident directions used in a quarter of a unit circle, which are the same for the observation directions. We consider the following directions (Figure 1(a))

$$
\theta_{j}=\hat{x}_{j}=(j-1) \frac{\pi}{2 N_{d}}, j=1,2, \ldots, 4 N_{d} .
$$

In the following examples, we consider $N_{d}=4$ and the point-like scatterers of the same scattering strength located at the points $y_{1}=(0,0), y_{2}=(0,0.5), y_{3}=(0.5,0), y_{4}=(0.5,0.5), y_{5}=(1,1), y_{6}=(1,-1), y_{7}=$ $(-1,-1), y_{8}=(-1,1), y_{9}=(1,-1.5), y_{10}=(1.5,0.5), y_{11}=(-1.5,1), y_{12}=(0,0.4), y_{13}=(0,-1), y_{14}=$ $(1.5,1.5)$ and $y_{15}=(0.6,0.6)$.

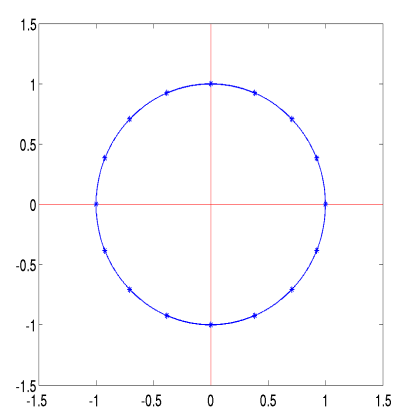

(a)

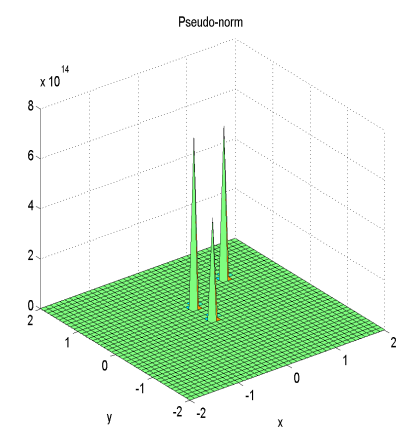

(b)

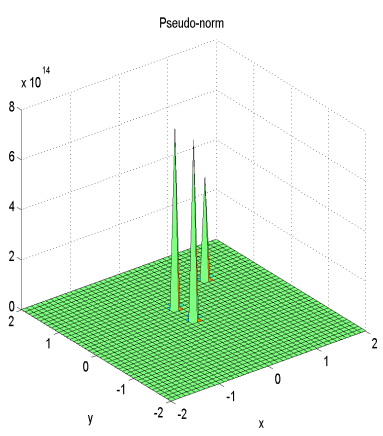

(c)

Figure 1: Incidence and observation directions with $N_{d}=4$ (a),

Born (b) and Foldy (c) based reconstructions with $0 \%$ noise, $g_{i}=1$ and $\kappa=\pi$ for 3 scatterers.

Since MUSIC algorithm is an exact method, the reconstruction is very accurate in the absence of noise in measured data, for both Born and Foldy models. It can be observed in figure 1(b-c), from the pseudo spectrum of the scatterers located at the points $y_{1}, y_{2}, y_{5}$ having scattering strengths 1 for each with the wavenumber $\kappa=\pi$ (i.e. minimum distance between the scatterers is quarter of the wavelength) with respect to the Born approximation and the Foldy model.

To analyze the effect of the noise level on the resolution of the algorithm, different noise levels are used. To distinguish the differences between the Born approximation and the Foldy model, we used different scattering strengths, noise levels and distance between the scatterers.

Figures 2 and 3 are related to the 8 scatterers located at the points $y_{1}, y_{2}, y_{5}, y_{6}, y_{7}, y_{8}, y_{10}$ and $y_{11}$ having scattering strength 1 for each with $5 \%$ random noise in the measured far field pattern. Figure 2 shows the pseudo spectrum of the mentioned 8 scatterers for the wavenumber $\kappa=2 \pi$ whereas figure 3 shows the pseudo spectrum for the wavenumber $\kappa=\pi$. We can observe that the scatterers satisfy largely the condition (2.21) and the reconstruction looks similar in both the Born approximation and the Foldy model. Hence, 


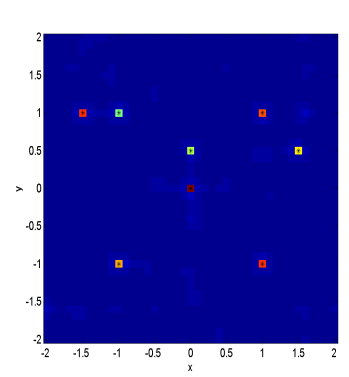

(a)

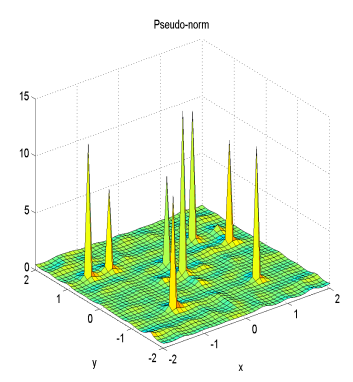

(b)

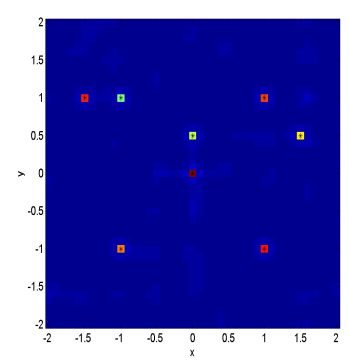

(c)

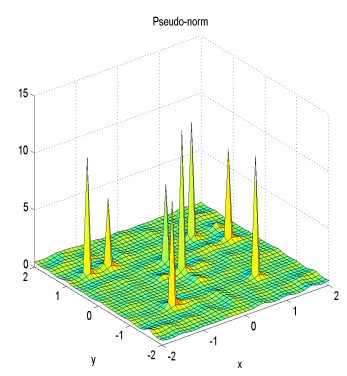

(d)

Figure 2: Born and Foldy based reconstructions respectively from left to right with $2 \%$ noise, $g_{i}=1$ and $\kappa=2 \pi$ for 8 scatterers.

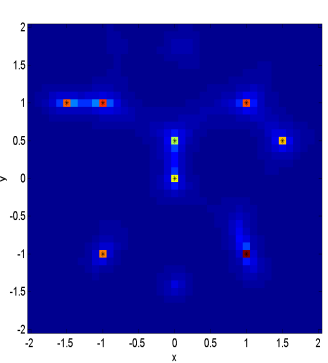

(a)

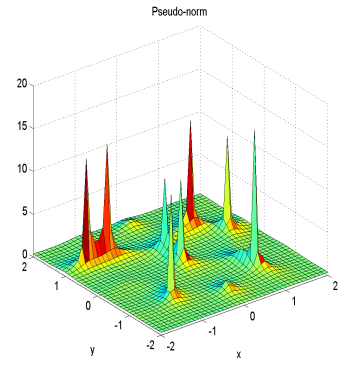

(b)

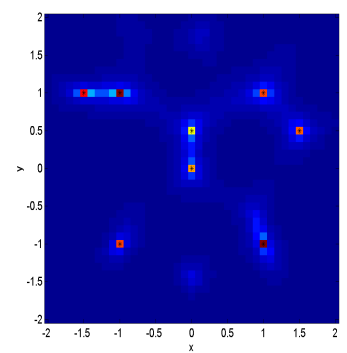

(c)

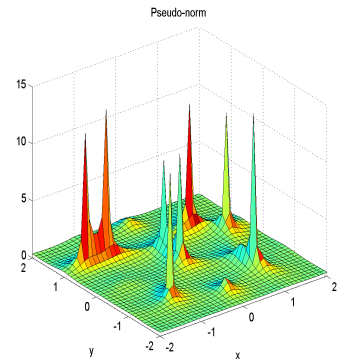

(d)

Figure 3: Born and Foldy based reconstructions respectively from left to right with $2 \%$ noise, $g_{i}=1$ and $\kappa=\pi$ for 8 scatterers.

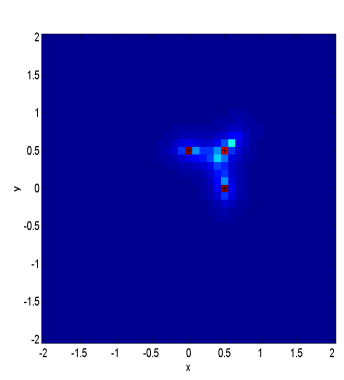

(a)

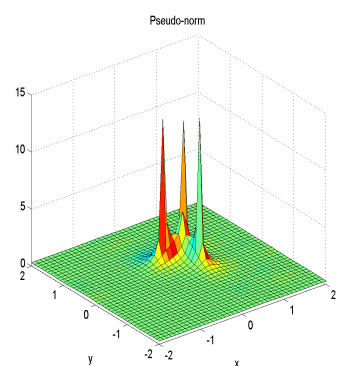

(b)

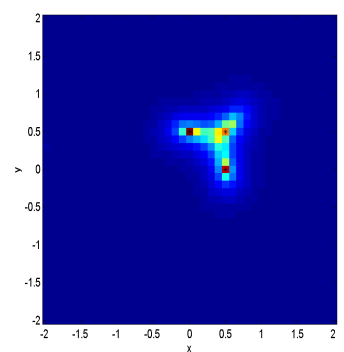

(c)

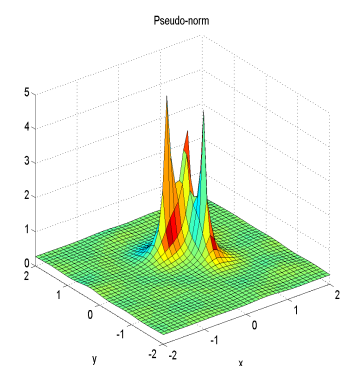

(d)

Figure 4: Born and Foldy based reconstructions respectively from left to right- $5 \%$ noise, $g_{i}=10$ and $\kappa=\pi$ for 3 scatterers. 


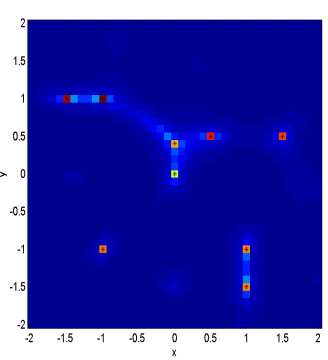

(a)

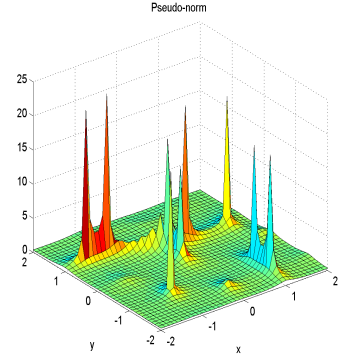

(b)

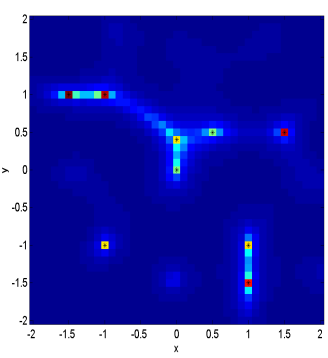

(c)

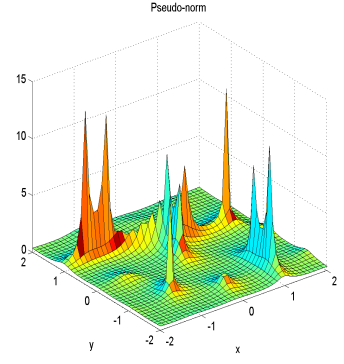

(d)

Figure 5: Born and Foldy based reconstructions respectively from left to right- $1 \%$ noise, $g_{i}=10$ and $\kappa=\pi$ for 9 scatterers.

we observe that if the scatterers are well separated with low scattering strengths there is no much difference in the reconstruction between the Born approximation and the Foldy model.

Now, we look for some examples where scatterers failed to satisfy the condition (2.21). Figure 4 shows the pseudo spectrum of the 3 scatterers located at $y_{2}, y_{3}$ and $y_{4}$ of each having scattering strength 10 for $\kappa=\pi$ with $5 \%$ random noise in the measured far field patterns with respect to the Born approximation and the Foldy method. Figure 5 shows the pseudo spectrum of the 9 scatterers located at $y_{1}, y_{4}, y_{6}, y_{7}, y_{8}, y_{9}, y_{10}, y_{11}$ and $y_{12}$ of each having scattering strength 10 for $\kappa=\pi$ with $1 \%$ random noise in the measured far field patterns with respect to the Born approximation and the Foldy model.

Compared to figures 2 and 3, we see in figure 4 and figure 5 how the reconstruction deteriorates due to the effect of multiple scattering created by the close obstacles. In this case, we can see the differences between the Born approximation and the Foldy model.

As a conclusion, we have seen that if the condition (2.21) is satisfied largely then the effect of the multiple scattering is quite low and the reconstruction is similar in both Born and Foldy but above the condition (2.21) the use of the Born approximation gives better reconstruction than the use of the Foldy method. However in the latter case, Born approximation is not valid as the scatterers are relatively close. It is observed that, in general, increase of the noise level, decrease of the distance between the scatterers and increase in the number of scatterers make the reconstruction worse in both the approximations in the presence of noise. It is also observed that when the scatterers have different scattering strengths and if they are not well separated, the visibility of the scatterer is proportional to the scattering strength of the respective scatterer.

\subsection{The inverse problem for the intermediate levels of scattering}

In this section, we deal with the intermediate levels of scattering. Recall that $U_{k}^{s}$, the scattered fields in the $k^{\text {th }}$ level scattering, has the form

$$
U_{k}^{s}(x)=\sum_{m=1}^{M} g_{m} \Phi\left(x, y_{m}\right) U_{m, k}\left(y_{m}\right)
$$

with the terms, exciting field, $U_{m, k}\left(y_{m}\right)$ are defined recursively by

$$
U_{m, k+1}\left(y_{m}\right):=U^{i}\left(y_{m}\right)+\sum_{\substack{j=1 \\ j \neq m}}^{M} g_{j} \Phi\left(y_{m}, y_{j}\right) U_{j, k}\left(y_{j}\right) \text { for } m=1, \ldots, M .
$$




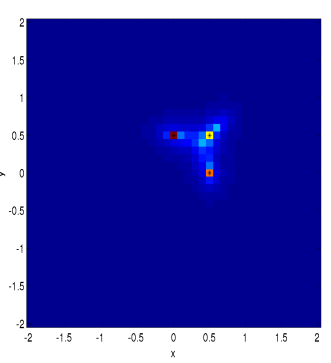

(a)

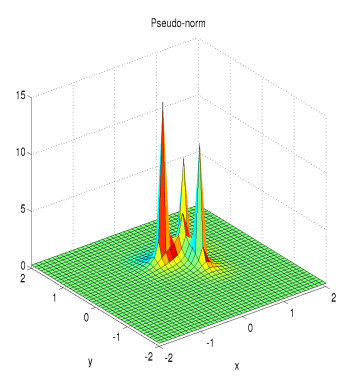

(b)

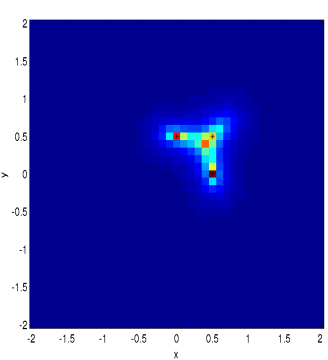

(c)

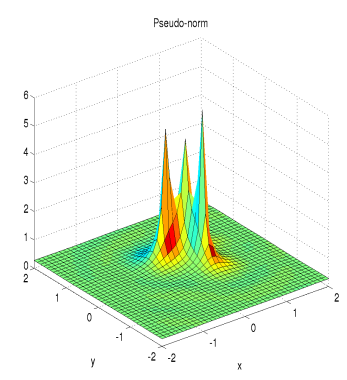

(d)

Figure 6: Figures are related to 3 scatterers with $5 \%$ noise, $g_{i}=10$ and $\kappa=\pi$. (a),(b)- $1^{\text {st }}$ level scattering, (c),(d)-3 $3^{\text {rd }}$ level scattering.

Define the vector $\mathbf{U}_{I, k} \in \mathbb{C}^{M}$ with components $U_{m, k}\left(y_{m}\right)$. Now, recall the definition of $\mathbf{U}^{I} \in \mathbb{C}^{M}$ in Section 2.3.1, then the exciting fields in different levels of scattering can be calculated from the below,

$$
\mathbf{U}_{I, k}=\sum_{l=0}^{k}(-\mathbb{M})^{l} \mathbf{U}^{I} \text { for } k=0,1, \ldots
$$

where the matrix $\mathbb{M} \in \mathbb{C}^{M \times M}$ is defined by $\mathbb{M}_{p q}:=-g_{q} \Phi\left(y_{p}, y_{q}\right)$ for $p \neq q$ and $\mathbb{M}_{p p}:=0 .^{2}$ Then using (2.15), the response matrix in the $k^{\text {th }}$ level scattering can be factorized as

$$
F=H^{*} T H,
$$

with $T:=\mathbf{g} \sum_{l=0}^{k}(-\mathbb{M})^{l}$, where $\mathbf{g}$ and $\mathrm{H}$ are defined in Section 2.3.1. To apply the MUSIC algorithm, the invertibility of the matrix $T$ is needed and the norm of $\mathbb{M}$ less than half is the sufficient condition for that in every level of scattering. In this case the reconstruction looks quite similar in all levels of scattering when the scatterers are far enough from each other. We can observe the similar kind of differences which we mentioned between weak (Born) and multiple (Foldy) scatterings, as the level $k$ of the scattering increases, with respect to the condition (2.21). We can observe this in figure 6 , for the same data as in figure 4 .

The more the condition $(2.21)$ is satisfied (i.e. L.H.S $\ll$ R.H.S) by the scatterers the more multiple scattering can be neglected which leads to weak scattering, i.e. reconstruction looks similar in various levels of scattering. But once this condition is violated, we see a clear difference between the reconstruction in different levels of scattering.

As discussed in Section 2.3.3, we can recover the matrix $T \in \mathbb{C}^{M \times M}$ as $T=I_{H} H F H^{*} I_{H}$, where $F$ is the given far field pattern in the $k^{\text {th }}$ level scattering and $H$ and $I_{H}$ are as mentioned earlier in Sections 2.3.1 and 2.3.3 respectively. By comparing this evaluated $T$ with its explicit form, $\mathbf{g} \sum_{l=0}^{k}(-\mathbb{M})^{l}$, in $k^{\text {th }}$ level scattering we can recover the scattering strengths $g_{m}$ 's. In Born and Foldy models it is clear as mentioned in Section 2.3.3 for $M$ scatterers. In the case $k=1$, we have $T=\mathbf{g}-\mathbf{g M}$. As we know that $\mathbf{g}$ is a diagonal matrix and the diagonal entries of $\mathbb{M}$ are zero, the diagonal entries of $T$ are equal to the scattering strengths $g_{1}, g_{2}, \cdots, g_{M}$ of the $M$ scatterers. But, for intermediate level scattering $k>1$, it is difficult to recover the scattering strengths due to the complicated structure of the matrices $(-\mathbb{M})^{l}$, for $l=2, \ldots$, and hence

\footnotetext{
${ }^{2}$ Observe that $(\mathbf{I}+\mathbb{M})=A$ which we mentioned in (2.19) of Section 2.3.1 for the Foldy case. Its inverse can be approximated by the truncated Neumann series in the case that the norm of $\mathbb{M}$ is less than one and, in case of 1-norm of $\mathbb{M}$, it is equal to the invertibility condition of $\mathcal{A}$ in Case 1 of Section 2.3.2.
} 
of $T$. For this reason, we restrict ourselves to the special case of two point-like obstacles $y_{1}, y_{2}$ with the corresponding scattering strengths $g_{1}, g_{2}$. In this case, we have the explicit form of $(-\mathbb{M})^{l}$ for $l=0,1,2, \ldots$ as follows

$$
(-\mathbb{M})^{l}=\left\{\begin{array}{cc}
{\left[\begin{array}{cc}
g_{1}^{\frac{l}{2}} g_{2}^{\frac{l}{2}} \Phi^{l}\left(y_{1}, y_{2}\right) & 0 \\
0 & g_{1}^{\frac{l}{2}} g_{2}^{\frac{l}{2}} \Phi^{l}\left(y_{1}, y_{2}\right)
\end{array}\right],} & l \in 2 \mathbb{N} \cup\{0\} \\
{\left[\begin{array}{cc}
0 & g_{1}^{\frac{l-1}{2}} g_{2}^{\frac{l+1}{2}} \Phi^{l}\left(y_{1}, y_{2}\right) \\
g_{1}^{\frac{l+1}{2}} g_{2}^{\frac{l-1}{2}} \Phi^{l}\left(y_{1}, y_{2}\right) & 0
\end{array}\right], \quad l \in 2 \mathbb{N}-1 .}
\end{array}\right.
$$

The matrix $(-\mathbb{M})^{l}$ is either diagonal or anti-diagonal for every $l \in \mathbb{N} \cup\{0\}$. This structure is not valid anymore for the case of more than two scatterers. From this structure, we obtain the explicit form of $T=\mathbf{g} \sum_{l=0}^{k}(-\mathbb{M})^{l}$ in the $k^{t h}$ order scattering as follows

$$
T=\left\{\begin{array}{cc}
{\left[\begin{array}{cc}
g_{1} & 0 \\
0 & g_{2}
\end{array}\right],} & k=0, \\
{\left[\begin{array}{cc}
g_{1} \sum_{l=0}^{\frac{k}{2}} g_{1}^{l} g_{2}^{l} \Phi^{2 l}\left(y_{1}, y_{2}\right) & \sum_{l=1}^{\frac{k}{2}} g_{1}^{l} g_{2}^{l} \Phi^{2 l-1}\left(y_{1}, y_{2}\right) \\
\sum_{l=1}^{\frac{k}{2}} g_{1}^{l} g_{2}^{l} \Phi^{2 l-1}\left(y_{1}, y_{2}\right) & g_{2} \sum_{l=0}^{\frac{k}{2}} g_{1}^{l} g_{2}^{l} \Phi^{2 l}\left(y_{1}, y_{2}\right)
\end{array}\right],} & k \in 2 \mathbb{N}, \\
{\left[\begin{array}{cc}
g_{1} \sum_{l=0}^{\frac{k-1}{2}} g_{1}^{l} g_{2}^{l} \Phi^{2 l}\left(y_{1}, y_{2}\right) & \sum_{l=0}^{\frac{k-1}{2}} g_{1}^{l+1} g_{2}^{l+1} \Phi^{2 l+1}\left(y_{1}, y_{2}\right) \\
\frac{k-1}{2} g_{1}^{l+1} g_{2}^{l+1} \Phi^{2 l+1}\left(y_{1}, y_{2}\right) & g_{2} \sum_{l=0}^{\frac{k-1}{2}} g_{1}^{l} g_{2}^{l} \Phi^{2 l}\left(y_{1}, y_{2}\right)
\end{array}\right], \quad k \in 2 \mathbb{N}-1,} \\
\frac{1}{g_{1}} & -\Phi\left(y_{1}, y_{2}\right) \\
-\Phi\left(y_{1}, y_{2}\right) & \frac{1}{g_{2}}
\end{array}\right]^{-1}, \quad k=\infty .
$$

From the above explicit form of $T$, we observe the following points.

- The diagonal entries of $T$ give the scattering strengths in the Born approximation, i.e. $k=0$.

- Substituting the non diagonal entries in the diagonal entries give the scattering strengths in every even level scattering $k$, i.e. $k \in 2 \mathbb{N}$. Indeed, define $a:=\sum_{l=1}^{\frac{k}{2}} g_{1}^{l} g_{2}^{l} \Phi^{2 l-1}\left(y_{1}, y_{2}\right)$ then the non-diagonal entries of $T$ are equal to $a$. Also the diagonal entries $T_{11}$ and $T_{22}$ of $T$ are equal to $g_{1}\left(1+\Phi\left(y_{1}, y_{2}\right) a\right)$ and $g_{2}\left(1+\Phi\left(y_{1}, y_{2}\right) a\right)$ respectively. Now, with the knowledge of the scatterers $y_{1}$ and $y_{2}$ from the MUSIC algorithm and by substituting the value of $a$ in the diagonal entries, we can evaluate the scattering strengths $g_{1}$ and $g_{2}$.

- Substituting the diagonal entries in the non diagonal entries give the scattering strengths in every odd level scattering $k$, i.e. $k \in 2 \mathbb{N}-1$. Indeed, define $b_{1}:=g_{1} \sum_{l=0}^{\frac{k-1}{2}} g_{1}^{l} g_{2}^{l} \Phi^{2 l}\left(y_{1}, y_{2}\right)$ and $b_{2}:=$ 
$g_{2} \sum_{l=0}^{\frac{k-1}{2}} g_{1}^{l} g_{2}^{l} \Phi^{2 l}\left(y_{1}, y_{2}\right)$ then the diagonal entries $T_{11}$ and $T_{22}$ of $T$ are equal to $b_{1}$ and $b_{2}$ respectively. Also the non-diagonal entries $T_{12}$ and $T_{21}$ of $T$ are the same and are equal to $g_{1} b_{2} \Phi\left(y_{1}, y_{2}\right)=g_{2} b_{1} \Phi\left(y_{1}, y_{2}\right)$. Now again with the knowledge of the scatterers $y_{1}$ and $y_{2}$ from the MUSIC algorithm and by substituting the diagonal entries in the non diagonal entries of $T$, we can evaluate the scattering strengths $g_{1}$ and $g_{2}$.

- The diagonal entries of $T^{-1}$ give the scattering strengths in the method of Foldy. i.e. $k=\infty$.

\section{$3 \quad$ Elastic scattering by point-like scatterers}

Assume that the Lamé coefficients $\lambda$ and $\mu$ are constants satisfying $\mu>0$ and $d \lambda+2 \mu>0, d=2,3$. We denote by $\rho$ a bounded and measurable density function such that $\rho=1$ outside of the bounded domain $D \subset \mathbb{R}^{d}$. The inhomogeneous problem associated to the Lamé system reads as follows,

$$
\left(\Delta^{e}+\omega^{2} \rho\right) u^{t}=0 \text { in } \mathbb{R}^{d}, \quad\left[\Delta^{e}:=\mu \Delta+(\lambda+\mu) \nabla \operatorname{div}\right]
$$

with the Kupradze radiation conditions

$$
\lim _{|x| \rightarrow \infty}|x|^{\frac{d-1}{2}}\left(\frac{\partial u_{p}}{\partial|x|}-i \kappa_{p} u_{p}\right)=0, \text { and } \lim _{|x| \rightarrow \infty}|x|^{\frac{d-1}{2}}\left(\frac{\partial u_{s}}{\partial|x|}-i \kappa_{s} u_{s}\right)=0,
$$

where the two limits are uniform in all the directions $\hat{x}:=\frac{x}{|x|} \in \mathbb{S}^{d-1}$. Here, we denoted the total field by $u^{t}:=u^{i}+u$, the incident field by $u^{i}$ and the scattered field by $u$. Also, we denoted $u_{p}:=-\kappa_{p}^{-2} \nabla(\nabla \cdot u)$ to be the longitudinal (or the pressure or P) part of the field $u$ and $u_{s}:=-\kappa_{s}^{-2} \nabla \times(\nabla \times u)$ to be the transversal (or the shear or S) part of the field $u$ corresponding to the Helmholtz decomposition $u=u_{p}+u_{s}$. The constants $\kappa_{p}:=\frac{\omega}{\sqrt{\lambda+2 \mu}}$ and $\kappa_{s}:=\frac{\omega}{\sqrt{\mu}}$ are known as the longitudinal and transversal wavenumbers, respectively and $\omega$ is the frequency. The scattering problem (3.1) - (3.2) is well posed, see [21, 22] for instance. In addition, the scattered field $u$ satisfies the following asymptotic expansion at infinity,

$$
u(x):=\frac{e^{i \kappa_{p}|x|}}{|x|^{\frac{d-1}{2}}} u_{p}^{\infty}(\hat{x})+\frac{e^{i \kappa_{s}|x|}}{|x|^{\frac{d-1}{2}}} u_{s}^{\infty}(\hat{x})+O\left(\frac{1}{|x|^{\frac{d+1}{2}}}\right),|x| \rightarrow \infty
$$

uniformly in all directions $\hat{x} \in \mathbb{S}^{d-1}$. The longitudinal part of the far field, i.e. $u_{p}^{\infty}(\hat{x})$ is normal to $\mathbb{S}^{d-1}$ while the transversal part $u_{s}^{\infty}(\hat{x})$ is tangential to $\mathbb{S}^{d-1}$. Due to this property, they can be measured separately. Note that it is not necessarily true for near field measurements. As usual in scattering problems, we use plane waves as incident waves in this work. For the Lamé system, they have the following analytic forms

$$
u^{i, p}(x, \theta):=\theta e^{i \kappa_{p} \theta \cdot x} \text { and } u^{i, s}(x, \theta):=\theta^{\perp} e^{i \kappa_{s} \theta \cdot x},
$$

where $\theta^{\perp}$ is any direction in $\mathbb{S}^{d-1}$ perpendicular to $\theta$. Pressure incident waves are in the direction of $\theta$, whereas shear incident waves are in the direction of $\theta^{\perp}$. In the two dimensional case, the shear waves have only one direction. But in the three dimensional case, they have two orthogonal components called vertical and horizontal shear directions denoted by $\theta^{\perp_{v}}$ and $\theta^{\perp_{h}}$ respectively. So, $\theta^{\perp}=\alpha \theta^{\perp_{h}}+\beta \theta^{\perp_{v}}$ for arbitrary constants $\alpha$ and $\beta$. To give the explicit forms of $\theta^{\perp_{h}}$ and $\theta^{\perp_{v}}$, we recall the Euclidean basis $\left\{e_{1}, e_{2}, e_{3}\right\}$ where 
$e_{1}:=(1,0,0), e_{2}:=(0,1,0)$ and $e_{3}:=(0,0,1)$, write $\theta:=\left(\theta_{1}, \theta_{2}, \theta_{3}\right)$ and set $r^{2}:=\theta_{1}^{2}+\theta_{2}^{2}$. Let $\mathcal{R}_{3}$ be the rotation map transforming $\theta$ to $e_{3}$. Then in the basis $\left\{e_{1}, e_{2}, e_{3}\right\}, \mathcal{R}_{3}$ is given by the matrix

$$
\mathcal{R}_{3}(\theta)=\frac{1}{r^{2}}\left[\begin{array}{ccc}
\theta_{2}^{2}+\theta_{1}^{2} \theta_{3} & -\theta_{1} \theta_{2}\left(1-\theta_{3}\right) & -\theta_{1} r^{2} \\
-\theta_{1} \theta_{2}\left(1-\theta_{3}\right) & \theta_{1}^{2}+\theta_{2}^{2} \theta_{3} & -\theta_{2} r^{2} \\
\theta_{1} r^{2} & \theta_{2} r^{2} & \theta_{3} r^{2}
\end{array}\right]
$$

It satisfies $\mathcal{R}_{3}^{T} \mathcal{R}_{3}=I$ and $\mathcal{R}_{3}(\theta)=e_{3}$. Correspondingly, we write $\theta^{\perp_{h}}:=\mathcal{R}_{3}^{T}\left(e_{1}\right)$ and $\theta^{\perp_{v}}:=\mathcal{R}_{3}^{T}\left(e_{2}\right)$. These two directions represent the horizontal and the vertical directions of the shear wave and they are given by

$$
\theta^{\perp_{h}}=\frac{1}{r^{2}}\left(\theta_{2}^{2}+\theta_{1}^{2} \theta_{3}, \theta_{1} \theta_{2}\left(\theta_{3}-1\right),-r^{2} \theta_{1}\right), \quad \theta^{\perp_{v}}=\frac{1}{r^{2}}\left(\theta_{1} \theta_{2}\left(\theta_{3}-1\right), \theta_{1}^{2}+\theta_{2}^{2} \theta_{3},-r^{2} \theta_{2}\right) .
$$

As in the scalar case, by setting $q:=\rho-1$, multiplying the equation (3.1) with the fundamental tensor $G(x, y)$ of the Navier equation and doing integration using Betti's third identity, see [22], we obtain the following Lippmann-Schwinger equation

$$
u^{t}(x)=u^{i}(x)+\omega^{2} \int_{D} q(y) G(x, y) u^{t}(y) d y .
$$

Here, we recall the form of $G(x, y)$,

$$
G(x, y):=-\frac{1}{\mu} \frac{\nabla \nabla}{\kappa_{s}^{2}} \Phi_{\kappa_{p}}(x, y)+\frac{1}{\mu}\left(I+\frac{\nabla \nabla}{\kappa_{s}^{2}}\right) \Phi_{\kappa_{s}}(x, y)
$$

where the first and the second parts represents the P-part and the S-Part of $G(x, y)$ respectively and $\Phi_{\kappa_{p}}(x, y)$, $\Phi_{\kappa_{s}}(x, y)$ are given by

$$
\Phi_{\kappa_{p}}(x, y):=\left\{\begin{array}{cc}
\frac{i}{4} \mathbf{H}_{0}^{1}\left(\kappa_{p}|x-y|\right) & \text { in } 2 D \\
\frac{e^{i \kappa_{p}|x-y|}}{4 \pi|x-y|} & \text { in } 3 D
\end{array}, \quad \Phi_{\kappa_{s}}(x, y):=\left\{\begin{array}{cc}
\frac{i}{4} \mathbf{H}_{0}^{1}\left(\kappa_{s}|x-y|\right) & \text { in } 2 D \\
\frac{e^{i \kappa_{s}|x-y|}}{4 \pi|x-y|} & \text { in } 3 D
\end{array} .\right.\right.
$$

To describe the elastic scattering by $M$ point-like scatterers $y_{1}, y_{2}, \ldots, y_{M}, M \in \mathbb{N}$, as in the acoustic case, we take $q$ as $q(y):=\frac{1}{\omega^{2}} \sum_{m=1}^{M} g_{m} \delta\left(y-y_{m}\right), g_{m} \in \mathbb{R}, m=1, \ldots, M$. Here again $\delta$ is the Dirac measure, $g_{m}$ 's are scattering strengths of the point-like scatterers $y_{m}$ 's. Now, we can write equation (3.7) formally as

$$
u^{t}(x)=u^{i}(x)+\sum_{m=1}^{M} g_{m} G\left(x, y_{m}\right) u^{t}\left(y_{m}\right), x \neq y_{m}, m=1,2, \ldots, M .
$$

As in the scalar case, we cannot calculate $u^{t}\left(y_{m}\right), m=1,2, \ldots, M$, since we cannot evaluate (3.9) at $x=y_{m}$ due to the singularity of $G\left(x, y_{m}\right)$ at $x=y_{m}$. We discuss the Born approximation, the Foldy method and then the intermediate levels of scattering.

\subsection{Born approximation}

In the Born approximation, the total field $u^{t}\left(y_{m}\right)$ is replaced by the incident field $u^{i}\left(y_{m}\right)$ at the point-like scatterers $y_{m}, m=1, \ldots, M$, in equation (3.9). So, the total field can be written as

$$
u^{t}(x)=u^{i}(x)+\sum_{m=1}^{M} g_{m} G\left(x, y_{m}\right) u^{i}\left(y_{m}\right)
$$


and then the scattered field can be written in the following form

$$
u(x)=\sum_{m=1}^{M} g_{m} G\left(x, y_{m}\right) u^{i}\left(y_{m}\right)
$$

The asymptotic behavior of Green tensor at infinity is given as follows,

$$
G\left(x, y_{m}\right)=a_{p} \hat{x} \otimes \hat{x} \frac{e^{i \kappa_{p}|x|}}{|x|^{\frac{n-1}{2}}} e^{-i \kappa_{p} \hat{x} \cdot y_{m}}+a_{s}(I-\hat{x} \otimes \hat{x}) \frac{e^{i \kappa_{s}|x|}}{|x|^{\frac{n-1}{2}}} e^{-i \kappa_{s} \hat{x} \cdot y_{m}}+O\left(|x|^{-\frac{n+1}{2}}\right),
$$

with $\hat{x}=\frac{x}{|x|} \in \mathbb{S}^{d-1}$ and $I$ being the identity matrix in $\mathbb{R}^{d}, a_{p}=\frac{\kappa_{p}^{2}}{4 \pi \omega^{2}}$ and $a_{s}=\frac{\kappa_{s}^{2}}{4 \pi \omega^{2}}$, see [1] for instance.

It follows from $(3.3),(3.11)$ and (3.12) that the $\mathrm{P}$ and the $\mathrm{S}$ parts of the far field pattern associated with the incident wave $u^{i, p}$ are given by

$$
\begin{aligned}
& u_{p}^{\infty, p}(\hat{x}, \theta)=a_{p} \sum_{m=1}^{M} g_{m}(\hat{x} \otimes \hat{x}) \cdot \theta e^{i \kappa_{p} y_{m} \cdot(\theta-\hat{x})} \\
& u_{s}^{\infty, p}(\hat{x}, \theta)=a_{s} \sum_{m=1}^{M} g_{m}(I-\hat{x} \otimes \hat{x}) \cdot \theta e^{i \kappa_{p} y_{m} \cdot \theta} e^{-i \kappa_{s} y_{m} \cdot \hat{x}}
\end{aligned}
$$

Similarly, the $\mathrm{P}$ and the $\mathrm{S}$ parts of the parts of the far field pattern associated with $\mathrm{S}$ incident wave $u^{i, s}$ can be written as

$$
\begin{aligned}
& u_{p}^{\infty, s}(\hat{x}, \theta)=a_{p} \sum_{m=1}^{M} g_{m}(\hat{x} \otimes \hat{x}) \cdot \theta^{\perp} e^{i \kappa_{s} y_{m} \cdot \theta} e^{-i \kappa_{p} y_{m} \cdot \hat{x}} \\
& u_{s}^{\infty, s}(\hat{x}, \theta)=a_{s} \sum_{m=1}^{M} g_{m}(I-\hat{x} \otimes \hat{x}) \cdot \theta^{\perp} e^{i \kappa_{s} y_{m} \cdot(\theta-\hat{x})}
\end{aligned}
$$

\subsection{Foldy's method}

Now, consider the multiple elastic scattering between the point-like obstacles. Similarly as in the scalar case, in the method of Foldy, the total field $u^{t}(x)$ has the form

$$
u^{t}(x)=u^{i}(x)+\sum_{m=1}^{M} g_{m} G\left(x, y_{m}\right) u_{m}\left(y_{m}\right)
$$

where the terms $u_{m}\left(y_{m}\right)$ 's can be calculated from the Foldy algebraic system given by

$$
u_{m}\left(y_{m}\right)=u^{i}\left(y_{m}\right)+\sum_{\substack{j=1 \\ j \neq m}}^{M} g_{j} G\left(y_{m}, y_{j}\right) u_{j}\left(y_{j}\right), \forall m=1, \ldots, M .
$$

We can write the Foldy algebraic system (3.18) in a compact form as

$$
\tilde{A} \mathbf{u}_{I}=\mathbf{u}^{I}
$$

where the matrix $\tilde{A} \in \mathbb{C}^{d M \times d M}$ and the vectors $\mathbf{u}_{I}, \mathbf{u}^{I} \in \mathbb{C}^{d M}$ are given by

$$
\tilde{A}:=\left(\begin{array}{cccc}
I & -g_{2} G\left(y_{1}, y_{2}\right) & \ldots & -g_{M} G\left(y_{1}, y_{M}\right) \\
-g_{1} G\left(y_{2}, y_{1}\right) & I & \ldots & -g_{M} G\left(y_{2}, y_{M}\right) \\
\ldots & \ldots & \ldots & \ldots \\
-g_{1} G\left(y_{M}, y_{1}\right) & -g_{2} G\left(y_{M}, y_{2}\right) & \ldots & I
\end{array}\right)
$$




$$
\mathbf{u}_{I}:=\left(\begin{array}{c}
u_{1}\left(y_{1}\right) \\
u_{2}\left(y_{2}\right) \\
\ldots \\
u_{M}\left(y_{M}\right)
\end{array}\right) \text { and } \mathbf{u}^{I}:=\left(\begin{array}{c}
u^{i}\left(y_{1}\right) \\
u^{i}\left(y_{2}\right) \\
\ldots \\
u^{i}\left(y_{M}\right)
\end{array}\right) .
$$

We denote $\mathbf{u}^{I}$ by $\mathbf{u}^{I, p}$ for $\mathrm{P}$ incident waves and by $\mathbf{u}^{I, s}$ for $\mathrm{S}$ incident waves.

Here, we inserted the fundamental matrix $G$, the identity matrix $I$, the incident vectors $u^{i}$ and the Foldy terms $u_{m}$ elementwise. We suppose that $\tilde{A}$ is non-singular and denote its inverse by $\tilde{B}$. From (3.19), we obtain the following representation

$$
\mathbf{u}_{I}=\tilde{B} \mathbf{u}^{I} .
$$

For each $m=1,2, \ldots, M$, set $\tilde{B}_{m} \in \mathbb{C}^{d \times d M}$ as a submatrix of $\tilde{B}$ formed by the rows related to the Foldy term $u_{m}\left(y_{m}\right)$, i.e. $\tilde{B}_{m}$ is formed by $d$ consecutive rows, from $((m-1) d+1)^{\text {th }}$ row to $m d^{\text {th }}$ row of $\tilde{B}$. With this setting, we obtain the scattered field from (3.18) and (3.17) as

$$
u(x)=\sum_{m=1}^{M} g_{m} G\left(x, y_{m}\right) u_{m}\left(y_{m}\right)=\sum_{m=1}^{M} g_{m} G\left(x, y_{m}\right) \tilde{B}_{m} \mathbf{u}^{I} .
$$

It follows from (3.3), (3.12) and (3.20) that the $\mathrm{P}$ and the $\mathrm{S}$ parts of the far field pattern associated with the $\mathrm{P}$ incident wave $u^{i, p}$ are given by

$$
\begin{array}{r}
u_{p}^{\infty, p}(\hat{x}, \theta)=a_{p} \sum_{m=1}^{M} g_{m}(\hat{x} \otimes \hat{x}) e^{-i \kappa_{p} \hat{x} \cdot y_{m}} \cdot \tilde{B}_{m} \cdot \mathbf{u}^{I, p}(\theta), \\
u_{s}^{\infty, p}(\hat{x}, \theta)=a_{s} \sum_{m=1}^{M} g_{m}(I-\hat{x} \otimes \hat{x}) e^{-i \kappa_{s} \hat{x} \cdot y_{m}} \cdot \tilde{B}_{m} \cdot \mathbf{u}^{I, p}(\theta) .
\end{array}
$$

Similarly, it follows from (3.3), (3.12) and (3.20) that the P and the S parts of the far field pattern associated with the $\mathrm{S}$ incident wave $u^{i, s}$ can be written as

$$
\begin{array}{r}
u_{p}^{\infty, s}(\hat{x}, \theta)=a_{p} \sum_{m=1}^{M} g_{m}(\hat{x} \otimes \hat{x}) e^{-i \kappa_{p} \hat{x} \cdot y_{m}} \cdot \tilde{B}_{m} \cdot \mathbf{u}^{I, s}(\theta), \\
u_{s}^{\infty, s}(\hat{x}, \theta)=a_{s} \sum_{m=1}^{M} g_{m}(I-\hat{x} \otimes \hat{x}) e^{-i \kappa_{s} \hat{x} \cdot y_{m}} \cdot \tilde{B}_{m} \cdot \mathbf{u}^{I, s}(\theta) .
\end{array}
$$

As we mentioned before, the P-parts of the far fields are normal to $\mathbb{S}^{d-1}$ and the S-parts are tangential. Using these properties, we define the following scalar far fields which will be useful in the statement and the justification of the MUSIC algorithm.

The scalar far field pattern associated with PP scattering ( $\mathrm{P}$ incident wave and $\mathrm{P}$ part of the far field) is

$$
u^{\infty}(\hat{x}, \theta):=\frac{\hat{x} \cdot u_{p}^{\infty, p}(\hat{x}, \theta)}{a_{p}}=\sum_{m=1}^{M} e^{-i \kappa_{p} \hat{x} \cdot y_{m}} g_{m}\left(\hat{x} \cdot \tilde{B}_{m} \cdot \mathbf{u}^{I, p}(\theta)\right) .
$$

The scalar far field pattern associated with SP scattering (S incident wave and P part of the far field) is

$$
u^{\infty}(\hat{x}, \theta):=\frac{\hat{x} \cdot u_{p}^{\infty, s}(\hat{x}, \theta)}{a_{p}}=\sum_{m=1}^{M} e^{-i \kappa_{p} \hat{x} \cdot y_{m}} g_{m}\left(\hat{x} \cdot \tilde{B}_{m} \cdot \mathbf{u}^{I, s}(\theta)\right)
$$


The scalar far field pattern associated with PS scattering (P incident wave and S part of the far field) is

$$
u^{\infty}(\hat{x}, \theta):=\frac{\hat{x}^{\perp} \cdot u_{s}^{\infty, p}(\hat{x}, \theta)}{a_{s}}=\sum_{m=1}^{M} e^{-i \kappa_{s} \hat{x} \cdot y_{m}} g_{m}\left(\hat{x}^{\perp} \cdot \tilde{B}_{m} \cdot \mathbf{u}^{I, p}(\theta)\right) .
$$

The scalar far field pattern associated with SS scattering (S incident wave and S part of the far field) is

$$
u^{\infty}(\hat{x}, \theta):=\frac{\hat{x}^{\perp} \cdot u_{s}^{\infty, s}(\hat{x}, \theta)}{a_{s}}=\sum_{m=1}^{M} e^{-i \kappa_{s} \hat{x} \cdot y_{m}} g_{m}\left(\hat{x}^{\perp} \cdot \tilde{B}_{m} \cdot \mathbf{u}^{I, s}(\theta)\right) .
$$

\subsection{Intermediate levels of scattering}

For any non negative integer $k$, let $u_{k}^{t}$ and $u_{k}^{s}$ denote the total and the scattered fields respectively in the $k^{\text {th }}$ level scattering. We set $u_{m, 0}=u^{i}, \forall m=1, \ldots, M$, to be the incident wave. Then the total field in the $k^{t h}$ level scattering is calculated by

$$
u_{k}^{t}(x)=u^{i}(x)+\sum_{m=1}^{M} g_{m} G\left(x, y_{m}\right) u_{m, k}\left(y_{m}\right),
$$

where the terms $u_{m, k}\left(y_{m}\right)$ 's, indicating the exciting fields, are defined recursively by

$$
u_{m, k+1}\left(y_{m}\right):=u^{i}\left(y_{m}\right)+\sum_{\substack{j=1 \\ j \neq m}}^{M} g_{j} G\left(y_{m}, y_{j}\right) u_{j, k}\left(y_{j}\right) \text { for } m=1, \ldots, M .
$$

The system (3.30) is nothing but the $k+1^{\text {th }}$ iteration of the Foldy algebraic system (3.18). Define the vector $\mathbf{u}_{I, k} \in \mathbb{C}^{d M}$ with components $u_{m, k}\left(y_{m}\right)$ arranged elementwise as in the pattern of $\mathbf{u}^{I}$ in Section 3.2. ${ }^{3}$ Recall the definitions of $\tilde{A} \in \mathbb{C}^{d M \times d M}, \mathbf{u}^{I} \in \mathbb{C}^{d M}$ in Section 3.2 and set $\tilde{\mathbb{M}}:=\tilde{A}-\mathbf{I},{ }^{4}$ then (3.30) can be written in a compact form as

$$
\mathbf{u}_{I, k}=\sum_{l=0}^{k}(-\tilde{\mathbb{M}})^{l} \mathbf{u}^{I} \text { for } k=0,1, \ldots
$$

Define the matrix $\tilde{C}_{k} \in \mathbb{C}^{d M \times d M}$ by $\tilde{C}_{k}:=\sum_{l=0}^{k}(-\tilde{\mathbb{M}})^{l}$ for $k=0,1, \ldots$. For each $m=1,2, \ldots, M$, set $\tilde{C}_{m, k} \in \mathbb{C}^{d \times d M}$ as a submatrix of $\tilde{C}_{k}$ formed by the rows related to the exciting field term $u_{m, k}\left(y_{m}\right)$, i.e. $\tilde{C}_{m, k}$ is formed by $d$ consecutive rows, from $((m-1) d+1)^{\text {th }}$ row to $m d^{\text {th }}$ row of $\tilde{C}_{k}$. With this setting and from (3.30), (3.29), we obtain the scattered field $u_{k}^{s}(x)$ in the $k^{\text {th }}$ level scattering as

$$
u_{k}^{s}(x)=\sum_{m=1}^{M} g_{m} G\left(x, y_{m}\right) u_{m, k}\left(y_{m}\right)=\sum_{m=1}^{M} g_{m} G\left(x, y_{m}\right) \tilde{C}_{m, k} \mathbf{u}^{I} .
$$

It follows from (3.3), (3.12) and (3.32) that the $\mathrm{P}$ and the $\mathrm{S}$ parts of the far field pattern in the $k^{\text {th }}$ level scattering associated with the $\mathrm{P}$ incident wave $u^{i, p}$ are given by

$$
\begin{array}{r}
u_{p, k}^{\infty, p}(\hat{x}, \theta)=a_{p} \sum_{m=1}^{M} g_{m}(\hat{x} \otimes \hat{x}) e^{-i \kappa_{p} \hat{x} \cdot y_{m}} \cdot \tilde{C}_{m, k} \cdot \mathbf{u}^{I, p}(\theta), \\
u_{s, k}^{\infty, p}(\hat{x}, \theta)=a_{s} \sum_{m=1}^{M} g_{m}(I-\hat{x} \otimes \hat{x}) e^{-i \kappa_{s} \hat{x} \cdot y_{m}} \cdot \tilde{C}_{m, k} \cdot \mathbf{u}^{I, p}(\theta) .
\end{array}
$$

\footnotetext{
${ }^{3}$ From (3.29) and (3.30), we can observe that $k=0 \& \infty$ deals with the Born approximation and the Foldy model respectively.

${ }^{4}$ In the case that the norm of $\mathbb{M}$ is less than one, the inverse of $\tilde{A}$ can be approximated by the truncated Neumann series.
} 
Similarly, it follows from (3.3), (3.12) and (3.32) that the P and the S parts of the far field pattern in the $k^{\text {th }}$ level scattering associated with the $\mathrm{S}$ incident wave $u^{i, s}$ can be written as

$$
\begin{array}{r}
u_{p, k}^{\infty, s}(\hat{x}, \theta)=a_{p} \sum_{m=1}^{M} g_{m}(\hat{x} \otimes \hat{x}) e^{-i \kappa_{p} \hat{x} \cdot y_{m}} \cdot \tilde{C}_{m, k} \cdot \mathbf{u}^{I, s}(\theta), \\
u_{s, k}^{\infty, s}(\hat{x}, \theta)=a_{s} \sum_{m=1}^{M} g_{m}(I-\hat{x} \otimes \hat{x}) e^{-i \kappa_{s} \hat{x} \cdot y_{m}} \cdot \tilde{C}_{m, k} \cdot \mathbf{u}^{I, s}(\theta) .
\end{array}
$$

As in the Foldy model, we define the following scalar versions of the far field patterns.

The scalar far field pattern in the $k^{\text {th }}$ level scattering associated with PP scattering (P incident wave and $\mathrm{P}$ part of the far field) is

$$
u_{k}^{\infty}(\hat{x}, \theta):=\frac{\hat{x} \cdot u_{p, k}^{\infty, p}(\hat{x}, \theta)}{a_{p}}=\sum_{m=1}^{M} e^{-i \kappa_{p} \hat{x} \cdot y_{m}} g_{m}\left(\hat{x} \cdot \tilde{C}_{m, k} \cdot \mathbf{u}^{I, p}(\theta)\right) .
$$

The scalar far field pattern in the $k^{\text {th }}$ level scattering associated with SP scattering (S incident wave and P part of the far field) is

$$
u_{k}^{\infty}(\hat{x}, \theta):=\frac{\hat{x} \cdot u_{p, k}^{\infty, s}(\hat{x}, \theta)}{a_{p}}=\sum_{m=1}^{M} e^{-i \kappa_{p} \hat{x} \cdot y_{m}} g_{m}\left(\hat{x} \cdot \tilde{C}_{m, k} \cdot \mathbf{u}^{I, s}(\theta)\right) .
$$

The scalar far field pattern in the $k^{\text {th }}$ level scattering associated with PS scattering (P incident wave and S part of the far field) is

$$
u_{k}^{\infty}(\hat{x}, \theta):=\frac{\hat{x}^{\perp} \cdot u_{s, k}^{\infty, p}(\hat{x}, \theta)}{a_{s}}=\sum_{m=1}^{M} e^{-i \kappa_{s} \hat{x} \cdot y_{m}} g_{m}\left(\hat{x}^{\perp} \cdot \tilde{C}_{m, k} \cdot \mathbf{u}^{I, p}(\theta)\right) .
$$

The scalar far field pattern in the $k^{\text {th }}$ level scattering associated with SS scattering (S incident wave and S part of the far field) is

$$
u_{k}^{\infty}(\hat{x}, \theta):=\frac{\hat{x}^{\perp} \cdot u_{s, k}^{\infty, s}(\hat{x}, \theta)}{a_{s}}=\sum_{m=1}^{M} e^{-i \kappa_{s} \hat{x} \cdot y_{m}} g_{m}\left(\hat{x}^{\perp} \cdot \tilde{C}_{m, k} \cdot \mathbf{u}^{I, s}(\theta)\right) .
$$

From the scalar far field patterns related to the Born approximation, Foldy model and intermediate level scatterings, we observe that there is no scattered field in the perpendicular directions ( i.e. $\hat{x} \perp \theta$ ) for PP and SS scatterings. Similarly, we observe that there is no scattered field in the parallel and anti parallel directions ( i.e. $\hat{x} \| \theta$ or $-\hat{x} \| \theta$ ) for PS and SP scatterings.

Inverse Problem : Given the far field pattern $u^{\infty}(\hat{x}, \theta)$, corresponding to each of the four situations described above for several incident and observation angles $\theta$ and $\hat{x}$, locate the point-like scatterers $y_{1}, y_{2}, \ldots, y_{M}$ and reconstruct the scattering strengths $g_{1}, g_{2}, \ldots, g_{M}$.

\subsection{The validity of the Born, Foldy and intermediate models}

Regarding the Born and the intermediate models, similar comments as in the scalar case can be made. The Foldy model (3.17)-(3.18) is justified in [18] where the scattering strengths $g_{m}$ are replaced by

$$
\left[g_{m}^{-1}-i \omega \frac{2 \lambda+5 \mu}{12 \pi \mu(\lambda+2 \mu)}\right]^{-1}
$$


for the $3 \mathrm{D}$ case and by

$$
\left[g_{m}^{-1}+\frac{1}{4 \pi}\left[\frac{\lambda+3 \mu}{\mu(\lambda+2 \mu)} C_{E}+\frac{\lambda+\mu}{\mu(\lambda+2 \mu)}-\frac{1}{2}\left(\frac{\ln \mu}{\mu}+\frac{\ln (\lambda+2 \mu)}{\lambda+2 \mu}\right)\right]\right]^{-1}
$$

in the $2 \mathrm{D}$ case, where $C_{E}$ is the Euler's constant. This extends the corresponding results in [2], known for the acoustic case, to the linearized isotropic elastic case.

\subsection{The inverse problems for the Foldy model}

Regarding the inverse problem for the Born model, we refer to [17] for the details. Let us mention that, there, it is shown that indeed only one type of elastic waves is enough for detecting the point-like scatterers, confirming the earlier results shown in [16] concerning extended scatterers. In the next sections, we deal with the Foldy and the intermediate models generalizing those results and provide a detailed study on the resolution of the reconstruction depending on the distance between the scatterers, the frequency used, the scattering strengths and the type of incident wave. This study could not be made in [17] since the Born model is not appropriate to analyze the resolution.

\subsubsection{MUSIC algorithm for elastic waves}

Here again we assume that the number of scatterers is not larger than the number of incident and observation directions, precisely $N \geq d M$. We define the response matrix $F \in \mathbb{C}^{N \times N}$ by

$$
\tilde{F}_{j l}:=U^{\infty}\left(\theta_{j}, \theta_{l}\right)
$$

In order to determine the locations $y_{m}$, we consider a grid of sampling points $z \in \mathbb{R}^{d}$. For each point $z$, we define the vectors $\phi_{z, p}^{j}$ and $\phi_{z, s}^{j}$ in $\mathbb{C}^{N}$ by

$$
\begin{aligned}
\phi_{z, p}^{j} & :=\left(\left(\theta_{1} \cdot e_{j}\right) e^{-i \kappa_{p} \theta_{1} \cdot z},\left(\theta_{2} \cdot e_{j}\right) e^{-i \kappa_{p} \theta_{2} \cdot z}, \ldots,\left(\theta_{N} \cdot e_{j}\right) e^{-i \kappa_{p} \theta_{N} \cdot z}\right)^{T}, \\
\phi_{z, s}^{j} & :=\left(\left(\theta_{1}^{\perp} \cdot e_{j}\right) e^{-i \kappa_{s} \theta_{1} \cdot z},\left(\theta_{2}^{\perp} \cdot e_{j}\right) e^{-i \kappa_{s} \theta_{2} \cdot z}, \ldots,\left(\theta_{N}^{\perp} \cdot e_{j}\right) e^{-i \kappa_{s} \theta_{N} \cdot z}\right)^{T}, \forall j=1, \ldots, d .
\end{aligned}
$$

In PP, PS, SS and SP scatterings, denote the response matrix $F$ by $F_{p}^{p}, F_{s}^{p}, F_{s}^{s}$ and $F_{p}^{s}$ respectively and these can be factorized as

$$
\begin{aligned}
F_{p}^{p} & =H^{p *} \tilde{\mathbf{g}} \tilde{B} H^{p}, \\
F_{s}^{p} & =H^{s *} \tilde{\mathbf{g}} \tilde{B} H^{p}, \\
F_{s}^{s} & =H^{s *} \tilde{\mathbf{g}} \tilde{B} H^{s}, \\
\text { and } F_{p}^{s} & =H^{p *} \tilde{\mathbf{g}} \tilde{B} H^{s} .
\end{aligned}
$$

Here, $\tilde{\mathbf{g}}:=\operatorname{Diag}\left(g_{1} I, g_{2} I, \ldots, g_{M} I\right) \in \mathbb{C}^{d M \times d M}$ is the diagonal matrix with diagonal entries as $g_{m}$ 's ( each $g_{m}$ appear $d$ times in $\tilde{\mathbf{g}}$ ) and the matrices $H^{p} \in \mathbb{C}^{d M \times N}$ and $H^{s} \in \mathbb{C}^{d M \times N}$ are defined as,

$$
H^{p}:=\left(\begin{array}{cccc}
\theta_{1} e^{i \kappa_{p} \theta_{1} \cdot y_{1}} & \theta_{2} e^{i \kappa_{p} \theta_{2} \cdot y_{1}} & \ldots & \theta_{N} e^{i \kappa_{p} \theta_{N} \cdot y_{1}} \\
\theta_{1} e^{i \kappa_{p} \theta_{1} \cdot y_{2}} & \theta_{2} e^{i \kappa_{p} \theta_{2} \cdot y_{2}} & \ldots & \theta_{N} e^{i \kappa_{p} \theta_{N} \cdot y_{2}} \\
\ldots & \ldots & \ldots & \ldots \\
\theta_{1} e^{i \kappa_{p} \theta_{1} \cdot y_{M}} & \theta_{2} e^{i \kappa_{p} \theta_{2} \cdot y_{M}} & \ldots & \theta_{N} e^{i \kappa_{p} \theta_{N} \cdot y_{M}}
\end{array}\right)
$$


and

$$
H^{s}:=\left(\begin{array}{cccc}
\theta_{1}^{\perp} e^{i \kappa_{s} \theta_{1} \cdot y_{1}} & \theta_{2}^{\perp} e^{i \kappa_{s} \theta_{2} \cdot y_{1}} & \ldots & \theta_{N}^{\perp} e^{i \kappa_{s} \theta_{N} \cdot y_{1}} \\
\theta_{1}^{\perp} e^{i \kappa_{s} \theta_{1} \cdot y_{2}} & \theta_{2}^{\perp} e^{i \kappa_{s} \theta_{2} \cdot y_{2}} & \ldots & \theta_{N}^{\perp} e^{i \kappa_{s} \theta_{N} \cdot y_{2}} \\
\ldots & \ldots & \ldots & \ldots \\
\theta_{1}^{\perp} e^{i \kappa_{s} \theta_{1} \cdot y_{M}} & \theta_{2}^{\perp} e^{i \kappa_{s} \theta_{2} \cdot y_{M}} & \ldots & \theta_{N}^{\perp} e^{i \kappa_{s} \theta_{N} \cdot y_{M}}
\end{array}\right)
$$

We already assumed that $\tilde{A} \in \mathbb{C}^{d M \times d M}$ is invertible and its inverse is $\tilde{B} \in \mathbb{C}^{d M \times d M}$. We can observe that $\tilde{A}$ can be factorized as $\tilde{A}=\tilde{\mathcal{A}} \tilde{\mathbf{g}}$ with $\tilde{\mathcal{A}} \in \mathbb{C}^{d M \times d M}$ defined by

$$
\tilde{\mathcal{A}}:=\left(\begin{array}{cccc}
\frac{1}{g_{1}} I & -G\left(y_{1}, y_{2}\right) & \ldots & -G\left(y_{1}, y_{M}\right) \\
-G\left(y_{2}, y_{1}\right) & \frac{1}{g_{2}} I & \ldots & -G\left(y_{2}, y_{M}\right) \\
\ldots & \ldots & \ldots & \ldots \\
-G\left(y_{M}, y_{1}\right) & -G\left(y_{M}, y_{2}\right) & \ldots & \frac{1}{g_{M}} I
\end{array}\right) .
$$

So, the matrix $\tilde{A}$ is invertible if and only if $\tilde{\mathcal{A}}$ is invertible. As we assumed $\tilde{A}$ is invertible, the matrix $\tilde{\mathcal{A}}$ is invertible and we get $\tilde{\mathbf{g}} \tilde{B}=\tilde{\mathbf{g}} \tilde{A}^{-1}=\tilde{\mathbf{g}}(\tilde{\mathcal{A}} \tilde{\mathbf{g}})^{-1}=\tilde{\mathcal{A}}^{-1}$. This gives us the factorization of the response matrices in PP, PS, SS and SP scatterings as

$$
\begin{aligned}
F_{p}^{p} & =H^{p *} \tilde{\mathcal{A}}^{-1} H^{p}, \\
F_{s}^{p} & =H^{s *} \tilde{\mathcal{A}}^{-1} H^{p}, \\
F_{s}^{s} & =H^{s *} \tilde{\mathcal{A}}^{-1} H^{s}, \\
\text { and } F_{p}^{s} & =H^{p *} \tilde{\mathcal{A}}^{-1} H^{s} .
\end{aligned}
$$

Under the assumption that $\tilde{\mathcal{A}}$ is invertible, we have the following theorem to justify the MUSIC algorithm for elastic wave scattering.

Theorem 3.1. Let $\left\{\theta_{j}: j \in \mathbb{N}\right\} \subset \mathbb{S}^{d-1}$ be a countable set of directions such that any analytic function on $\mathbb{S}^{d-1}$ that vanishes in $\theta_{j}$ for all $j \in \mathbb{N}$ vanishes identically. Let $\mathbf{K}$ be a compact subset of $\mathbb{R}^{d}$ containing $\left\{y_{m}: m=1, \ldots, M\right\}$. Then there exists $N_{0} \in \mathbb{N}$ such that for any $N \geq N_{0}$ the following characterization holds for every $z \in \mathbf{K}$ :

$$
\begin{aligned}
z \in\left\{y_{1}, \ldots, y_{M}\right\} & \Longleftrightarrow \phi_{z, p}^{j} \in \mathcal{R}\left(H^{p^{*}}\right) \\
& \\
\phi_{z, s}^{j} \in \mathcal{R}\left(H^{s *}\right), & \text { for some } j=1, \ldots, d .
\end{aligned}
$$

Furthermore,

- The ranges of $H^{p^{*}}$ and $F_{p}$ (where, $F_{p}:=F_{p}^{p}$ or $\left.F_{p}:=F_{p}^{s}\right)$ coincide and thus

$$
z \in\left\{y_{1}, \ldots, y_{M}\right\} \Longleftrightarrow \phi_{z, p}^{j} \in \mathcal{R}\left(F_{p}\right) \Longleftrightarrow \mathcal{P}_{p} \phi_{z, p}^{j}=0 \text {, for some } j=1, \ldots, d,
$$

where $\mathcal{P}_{p}: \mathbb{C}^{N} \rightarrow \mathcal{R}\left(F_{p}\right)^{\perp}=\mathcal{N}\left(F_{p}{ }^{*}\right)$ is the orthogonal projection onto the null space $\mathcal{N}\left(F_{p}{ }^{*}\right)$ of $F_{p}{ }^{*}$.

- The ranges of $H^{s *}$ and $F_{s}$ (where, $F_{s}:=F_{s}^{p}$ or $\left.F_{s}:=F_{s}^{s}\right)$ coincide and thus

$$
z \in\left\{y_{1}, \ldots, y_{M}\right\} \Longleftrightarrow \phi_{z, s}^{j} \in \mathcal{R}\left(F_{s}\right) \Longleftrightarrow \mathcal{P}_{s} \phi_{z, s}^{j}=0, \text { for some } j=1, \ldots, d,
$$

where $\mathcal{P}_{s}: \mathbb{C}^{N} \rightarrow \mathcal{R}\left(F_{s}\right)^{\perp}=\mathcal{N}\left(F_{s}{ }^{*}\right)$ is the orthogonal projection onto the null space $\mathcal{N}\left(F_{s}{ }^{*}\right)$ of $F_{s}{ }^{*}$. 
Proof: The idea of the proof is essentially the same as the one of Theorem 4.1 in [20] concerning the acoustic case. The main task in proving this theorem is to show that the matrices $H^{s}$ and $H^{p}$ have maximal rank. For convenience of the reader, we give it in appendix since some technical difficulties have to be taken care of.

We can prove this theorem also for the different set of incident and the observational angles given by the assumption that " $\left\{\theta_{j}: j \in \mathbb{N}\right\} \subset \mathbb{S}^{d-1}$ and $\left\{x^{j}: j \in \mathbb{N}\right\} \subset \mathbb{S}^{d-1}$ are countable set of incident and observational directions such that any analytic function on $\mathbb{S}^{d-1}$ that vanishes on one of these sets, will vanish identically". From Theorem 3.1, the MUSIC algorithm holds for the response matrices corresponding to the PP, PS, SS and SP scatterings using Foldy method, under the assumption of the invertibility of the matrix $\tilde{\mathcal{A}}$. To make the best use of the singular value decomposition in SP and PS scatterings, we apply the MUSIC algorithm to $F_{p}^{s} F_{p}^{s *}\left(\operatorname{resp}, F_{p}^{s^{*}} F_{p}^{s}\right)$ and $F_{s}^{p^{*}} F_{s}^{p}\left(\operatorname{resp}, F_{s}^{p} F_{s}^{p^{*}}\right)$ with the help of the test vectors $\phi_{z, p}^{j}\left(\right.$ resp, $\left.\phi_{z, s}^{j}\right)$ respectively.

The point-like scatterers can then be located in the PP, PS, SS and SP scatterings of elastic waves in the Foldy regime. In addition, in the three dimensional case, while dealing with $\mathrm{S}$ incident wave or $\mathrm{S}$ part of the far field pattern, it is enough to use one of its horizontal $\left(\mathrm{S}^{h}\right)$ or vertical $\left(\mathrm{S}^{v}\right)$ parts. Hence, it is enough to study the far field pattern of any of the PP, $\mathrm{PS}^{h}, \mathrm{PS}^{v}, \mathrm{~S}^{h} \mathrm{~S}^{h}, \mathrm{~S}^{h} \mathrm{~S}^{v}, \mathrm{~S}^{v} \mathrm{~S}^{h}, \mathrm{~S}^{v} \mathrm{~S}^{v}, \mathrm{~S}^{h} \mathrm{P}, \mathrm{S}^{v} \mathrm{P}$ elastic scatterings to locate the scatterers. In other words, in three dimensional case, instead of using the full incident wave and the full far field pattern, it is enough to study one combination of pressure $(\mathrm{P})$, horizontal shear $\left(\mathrm{S}^{h}\right)$ or vertical shear $\left(\mathrm{S}^{v}\right)$ parts of the elastic incident wave and a corresponding part of the elastic far field patterns. Indeed, define the vectors $\phi_{z, s^{h}}^{j}, \phi_{z, s^{v}}^{j} \in \mathbb{C}^{N}$ and the matrices $H^{s^{h}}, H^{s^{v}} \in \mathbb{C}^{3 M \times N}$ exactly as $\phi_{z, s}^{j}$ and $H^{s}$ replacing $\theta_{i}^{\perp}$ for $i=1, \ldots, N$ by $\theta_{i}^{\perp_{h}}$ and $\theta_{i}^{\perp_{v}}$ respectively, see (3.6). We denote the response matrices by $F_{s^{h}}^{p}, F_{p}^{s^{h}}, F_{s^{v}}^{p}, F_{p}^{s^{v}}, F_{s^{h}}^{s^{h}}, F_{s^{v}}^{s^{h}}, F_{s^{h}}^{s^{v}}$, and $F_{s^{v}}^{s^{v}}$ in the elastic $\mathrm{PS}^{h}, \mathrm{~S}^{h} \mathrm{P}, \mathrm{PS}^{v}, \mathrm{~S}^{v} \mathrm{P}, \mathrm{S}^{h} \mathrm{~S}^{h}, \mathrm{~S}^{h} \mathrm{~S}^{v}, \mathrm{~S}^{v} \mathrm{~S}^{h}, \mathrm{~S}^{v} \mathrm{~S}^{v}$ scatterings respectively, then we can factorize them as follows

$$
\begin{aligned}
F_{s^{h}}^{p} & =H^{s^{h *}} \tilde{\mathcal{A}}^{-1} H^{p}, & F_{p}^{s^{h}} & =H^{p *} \tilde{\mathcal{A}}^{-1} H^{s^{h}}, \\
F_{s^{v}}^{p} & =H^{s^{v *}} \tilde{\mathcal{A}}^{-1} H^{p}, & F_{p}^{s^{v}} & =H^{p^{*}} \tilde{\mathcal{A}}^{-1} H^{s^{v}}, \\
F_{s^{h}}^{s^{h}} & =H^{s^{h *}} \tilde{\mathcal{A}}^{-1} H^{s^{h}}, & F_{s^{v}}^{s^{h}} & =H^{s^{* *}} \tilde{\mathcal{A}}^{-1} H^{s^{h}}, \\
\text { and } \quad F_{s^{h}}^{s^{v}} & =H^{s^{h *}} \tilde{\mathcal{A}}^{-1} H^{s^{v}}, & F_{s^{v}}^{s^{v}} & =H^{s^{v *}} \tilde{\mathcal{A}}^{-1} H^{s^{v}} .
\end{aligned}
$$

Hence in the 3D case, we can state the following theorem related to the MUSIC algorithm,

Theorem 3.2. Let $\left\{\theta_{j}: j \in \mathbb{N}\right\} \subset \mathbb{S}^{2}$ be a countable set of directions such that any analytic function on $\mathbb{S}^{2}$ that vanishes in $\theta_{j}$ for all $j \in \mathbb{N}$ vanishes identically. Let $\mathbf{K}$ be a compact subset of $\mathbb{R}^{3}$ containing $\left\{y_{m}: m=1, \ldots, M\right\}$. Then there exists $N_{0} \in \mathbb{N}$ such that for any $N \geq N_{0}$ the following characterization holds for every $z \in \mathbf{K}$ :

$$
z \in\left\{y_{1}, \ldots, y_{M}\right\} \Longleftrightarrow \phi_{z, t}^{j} \in \mathcal{R}\left(H^{t^{*}}\right), \text { for some } j=1,2,3 \text { and for all } t \in\left\{p, s^{h}, s^{v}\right\} .
$$

Furthermore, the ranges of $H^{t^{*}}$ and $F_{t}^{r}$ coincide and thus

$$
z \in\left\{y_{1}, \ldots, y_{M}\right\} \Longleftrightarrow \phi_{z, t}^{j} \in \mathcal{R}\left(F_{t}^{r}\right) \Longleftrightarrow \mathcal{P}_{t} \phi_{z, t}^{j}=0, \text { for some } j=1,2,3 \text { and for all } r, t \in\left\{p, s^{h}, s^{v}\right\}
$$

where $\mathcal{P}_{t}: \mathbb{C}^{N} \rightarrow \mathcal{R}\left(F_{t}^{r}\right)^{\perp}=\mathcal{N}\left(F_{t}^{r *}\right)$ is the orthogonal projection onto the null space $\mathcal{N}\left(F_{t}^{r *}\right)$ of $F_{t}^{r *}$.

Proof: The proof of this theorem is the same as the one of Theorem 3.1, by proving the maximal rank property of the matrices $H^{s^{h}}, H^{s^{v}}$ and $H^{p}$ and by using the test vectors $\phi_{z, p}^{j}, \phi_{z, s^{h}}^{j}$ and $\phi_{z, s^{v}}^{j}$, see the appendix for more details. 


\subsubsection{Invertibility of the matrix $\tilde{\mathcal{A}}$}

As in the scalar case, when the scatterers are relatively far away from each other comparing to the scattering strengths, then the invertibility condition of $\tilde{\mathcal{A}}$ is the diagonally dominant condition and it is given by

$$
\sum_{\substack{i=1 \\ i \neq j}}^{M}\left\|G\left(y_{i}, y_{j}\right)\right\|_{\infty}<\frac{1}{\left|g_{j}\right|}, \forall j=1,2, \ldots, M .
$$

Here $\|\cdot\|_{\infty}$ is the infinite norm and it is defined for a matrix, $\mathbf{L}=\left[L_{m n}\right] \in \mathbb{C}^{M \times N}$, as $\|\mathbf{L}\|_{\infty}:=$ $\max _{1 \leq m \leq M} \sum_{n=1}^{N}\left|L_{m n}\right|$. Recall the matrix $G(x, y)$ from (3.8). It can be written explicitly in 3D as

$$
\begin{aligned}
G(x, y)= & \frac{1}{\mu k_{s}^{2}} \frac{\Phi_{p}(x, y)}{r^{2}}\left[k_{p}^{2} R R+\left(1-i k_{p} r\right)(I-3 \hat{R} \hat{R})\right] \\
& -\frac{1}{\mu k_{s}^{2}} \frac{\Phi_{s}(x, y)}{r^{2}}\left[k_{s}^{2} R R+\left(1-i k_{s} r\right)(I-3 \hat{R} \hat{R})\right]+\frac{\Phi_{s}(x, y)}{\mu} I,
\end{aligned}
$$

where $R=x-y, r=|x-y|$ and $\hat{R}=\frac{R}{r}$, see [10]. Similarly, by writing the explicit form of $G(x, y)$ in 2D, we observe that it is expressed explicitly in terms of $x-y$. In (3.62), we remark that the entries of $G(x, y)$ are analytic in terms of the variables $\eta_{i j l}=\left(y_{i}-y_{j}\right)_{l}, i, j=1, \ldots, M$ and $l=1, \ldots, d$ for $\eta_{i j l} \in \mathbb{R} \backslash\{0\}$. Remark also that $\operatorname{det} \tilde{\mathcal{A}}$ is given by the products and sums of $g_{i}^{-1}$ and the entries of $G\left(y_{i}, y_{j}\right)$ for $i, j=1, \ldots, M$. From the above discussion, using the analyticity of the determinant of $\tilde{\mathcal{A}}$ in terms of the $d \frac{M(M-1)}{2}$ real variables $\eta_{i j l}$ for $i, j=1, \ldots, M$ with $i<j, l=1, \ldots, d$, fixing the frequency $\omega$ and the scattering strengths $g_{i}$, for $i=1, \ldots, M$, we can show that except for few distributions of the scatterers, $y_{1}, \ldots, y_{M}$, the matrix $\tilde{\mathcal{A}}$ is invertible.

\subsubsection{Recovering the scattering strengths $g_{m}$ 's}

We have the factorization of the response matrix in PP, PS and SP elastic scatterings using Foldy method as

$$
F_{t}^{r}=H^{t^{*}} \tilde{\mathcal{A}}^{-1} H^{r}, \forall r, t \in\{p, s\} .
$$

As in the acoustic case, once we locate the scatterers for the given scalar far field patterns, we can recover the scattering strengths from the factorization of $F_{t}^{r} \in \mathbb{C}^{N \times N}$. Indeed, from Theorem 3.1, we know that the matrices $H^{p}$ and $H^{s}$ have maximal rank. So, the matrices $H^{p} H^{p *} \in \mathbb{C}^{d M \times d M}$ and $H^{s} H^{s *} \in \mathbb{C}^{d M \times d M}$ are invertible. Let us denote these inverses by $I_{H^{p}}$ and $I_{H^{s}}$ respectively. Once we locate the scatterers $y_{1}, y_{2}, \ldots, y_{M}$ by using MUSIC algorithm for the given far field patterns, we can recover the matrix $\tilde{\mathcal{A}}^{-1} \in$ $C^{d M \times d M}$ as $\tilde{\mathcal{A}}^{-1}=I_{H^{t}} H^{t} F_{t}^{r} H^{r *} I_{H^{r}}, I_{H^{t}} H^{t}$ (resp, $\left.H^{r *} I_{H^{r}}\right)$ is the pseudo inverse of $H^{t^{*}}$ (resp, $H^{r}$ ). Then,

we can recover the scattering strengths $g_{1}, \ldots, g_{M}$ from the diagonal entries of $\tilde{\mathcal{A}}$. In the similar way, also in the 3D case we can recover the scattering strengths in the case of $\mathrm{PS}^{h}, \mathrm{~S}^{h} \mathrm{P}, \mathrm{PS}^{v}, \mathrm{~S}^{v} \mathrm{P}, \mathrm{S}^{h} \mathrm{~S}^{h}, \mathrm{~S}^{h} \mathrm{~S}^{v}, \mathrm{~S}^{v} \mathrm{~S}^{h}$ and $\mathrm{S}^{v} \mathrm{~S}^{v}$ elastic scatterings for $r, t \in\left\{p, s^{h}, s^{v}\right\}$.

\subsubsection{Numerical results and discussions}

In this section, we illustrate the performance of the MUSIC algorithm for the elastic waves for locating the scatterers using Foldy method. We present the results comparing the weak (Born) and multiple (Foldy) 
scatterings using one type of wave. In addition, we compare the results for the case of S and P incident plane waves.

Here also, we only show results for two dimensional problems. From the previous sections, we know that one type of transverse wave is sufficient to locate the scatterers in three dimensional case. Denote by $N_{d}$, the number of incident directions used in a quarter of a unit circle which are same for the observational directions. There are no restrictions on incident and observational angles but there are some points one should consider:

(1) It is better to avoid perpendicular directions for $\mathrm{P}$ incident waves and $\mathrm{P}$ part of far field patterns (PP case), or S incident waves and S part of far field patterns (SS case) as they dont provide any useful information due to no scattered far field in these directions. To avoid the perpendicular directions, in the first and the third quarters, we use the following incidence angles (see Figure 7(a))

$$
\begin{aligned}
\theta_{j} & =(j-1) \frac{\pi}{2 N_{d}}, \\
\theta_{2 N_{d}+j} & =\pi+(j-1) \frac{\pi}{2 N_{d}}, j=1, \ldots, N_{d},
\end{aligned}
$$

and in the second and the fourth quarters, we make use of the incidence angles

$$
\begin{aligned}
\theta_{N_{d}+j} & =\frac{\pi}{2}+\frac{\pi}{4 N_{d}}+(j-1) \frac{\pi}{2 N_{d}}, \\
\theta_{3 N_{d}+j} & =\frac{3 \pi}{2}+\frac{\pi}{4 N_{d}}+(j-1) \frac{\pi}{2 N_{d}}, j=1, \ldots, N_{d} .
\end{aligned}
$$

The observation directions are taken the same as the incidence one. In this setup, we have $|\hat{x} \cdot \theta| \geq \sin \left(\frac{\pi}{4 N_{d}}\right)$ for all $\hat{x}, \theta \in\left\{\theta_{j}, j=1, \ldots, 4 N_{d}\right\}$.

(2) It is better to avoid parallel or anti-parallel directions in the case of P incident waves and S part of far field patterns (PS case) or S incident waves and P part of far field patterns (SP case) as they dont provide any useful information due to no scattered far field in these directions. To avoid this, we choose the incident and observation angles as follows (Figure 7(b)).

$$
\begin{aligned}
\theta_{j} & =(j-1) \frac{\pi}{2 N_{d}}, j=1, \ldots, 4 N_{d}, \\
\hat{x}_{j} & =\theta_{j}+\frac{\pi}{4 N_{d}}, j=1, \ldots, 4 N_{d} .
\end{aligned}
$$

With this choice, the minimum angle between the incidence and observation angles is $\frac{\pi}{4 N_{d}}$.

To show the differences between the Born approximation and the Foldy model, we used different scattering strengths, noise levels and distance between the scatterers. We observed the similar kind of variations between the Born approximation and the Foldy model which are mentioned in the case of acoustic scattering in Section 2.3.4. It is important to mention that, by converting the vector far field patterns to the scalar ones as in equation (3.25), the noise in the measured far field patterns is amplified in the modified multistatic response matrix resulting worse results than the scalar case. In the following examples, the parameters are chosen as $\lambda=1$ and $\mu=1$ resulting in $\kappa_{p}=\kappa / \sqrt{3}$ and $\kappa_{s}=\kappa$. Let us consider the points $y_{2}, y_{3}, y_{4}, y_{5}, y_{6}, y_{7}, y_{8}, y_{13}$ and $y_{14}$ which we mentioned in Section 2.3.4. We have chosen $N_{d}=4$, which gives us the total number of incident directions as $4 N_{d}=16$.

Figures 8 and 10 are related to three scatterers located at the points $y_{2}, y_{5}$ and $y_{13}$. In figures 8 and 10 , each scatterer is of scattering strength 1 for the wavenumber $\kappa=\pi$ with $2 \%$ random noise in the measured far field patterns. Figure 9 is related to six scatterers located at the points $y_{2}, y_{5}, y_{6}, y_{7}, y_{8}$ and $y_{13}$ of 


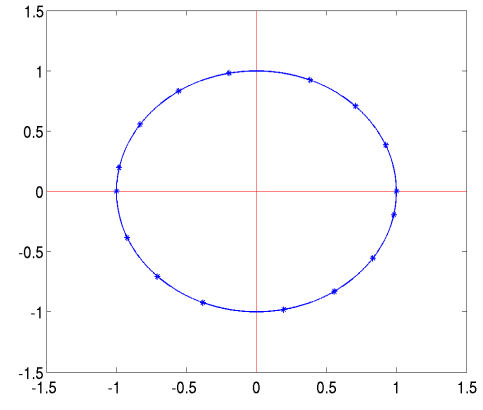

(a)

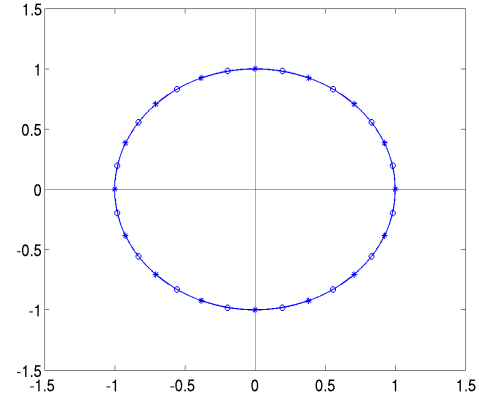

(b)

Figure 7: Incidence and observation directions with $N_{d}=4$ : (a) PP and SS cases (the incidence and observations coincide); (b) PS and SP cases ('*': incidence directions, 'o': observation directions)

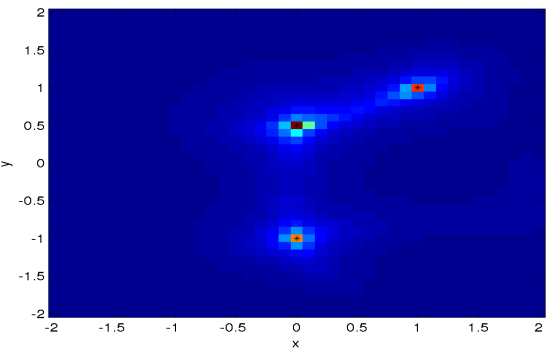

(a)

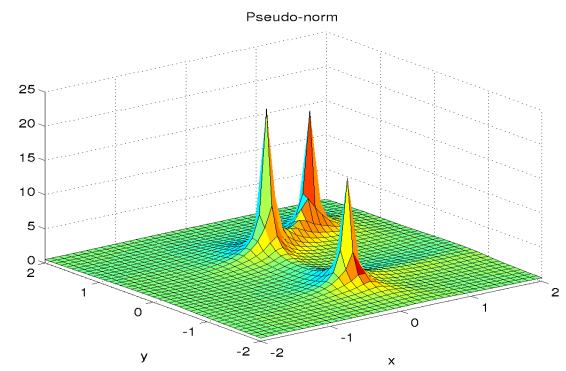

(c)

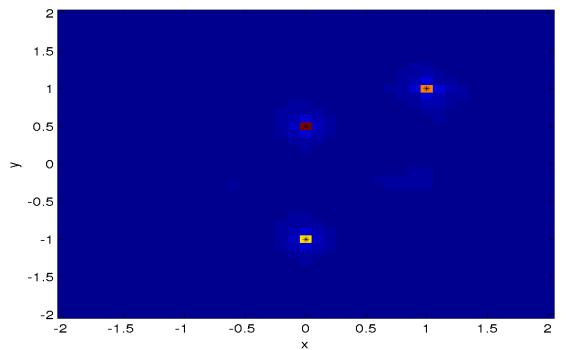

(b)

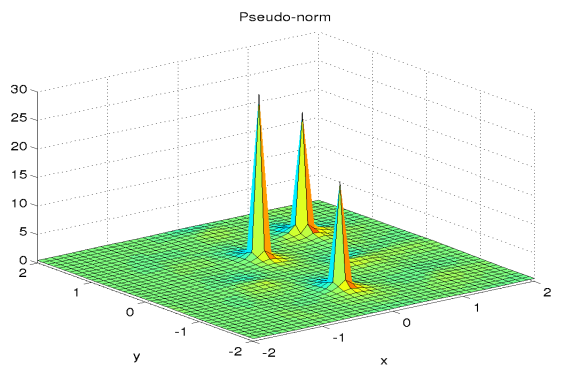

(d)

Figure 8: Multiple(Foldy) PP and SP scatterings respectively from left to right with $2 \%$ noise, $g_{i}=1$ and $\kappa=\pi$ for 3 scatterers. 


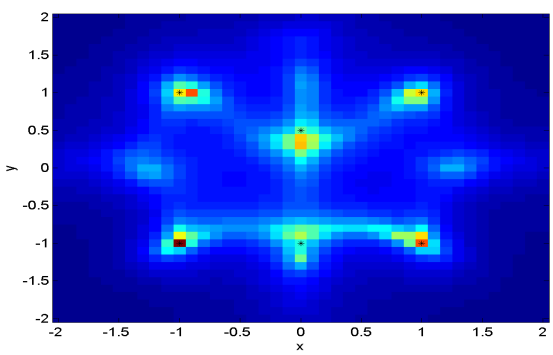

(a)

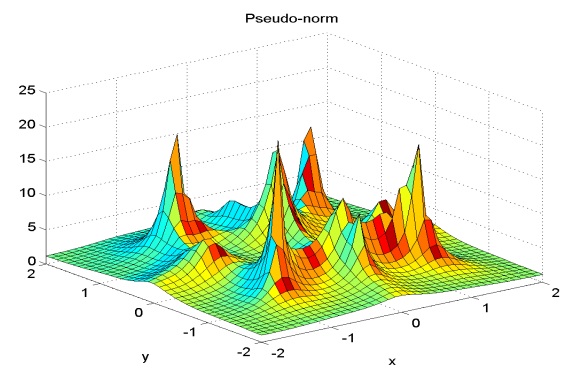

(c)

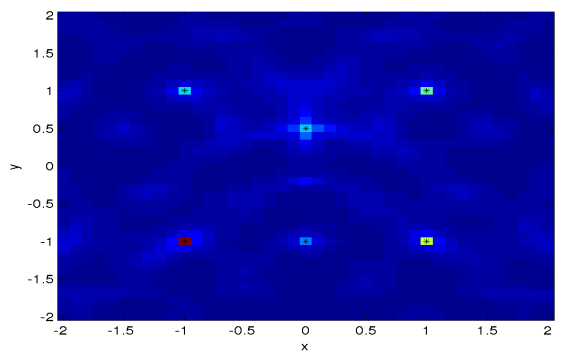

(b)

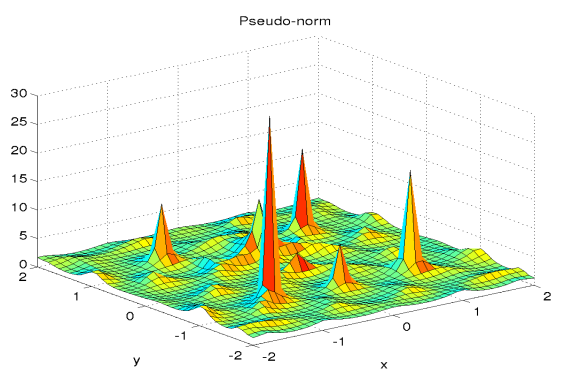

(d)

Figure 9: Multiple(Foldy) PP and SP scatterings respectively from left to right with $2 \%$ random noise, $g_{i}=1$ and $\kappa=1.5 \pi$ for 6 scatterers.

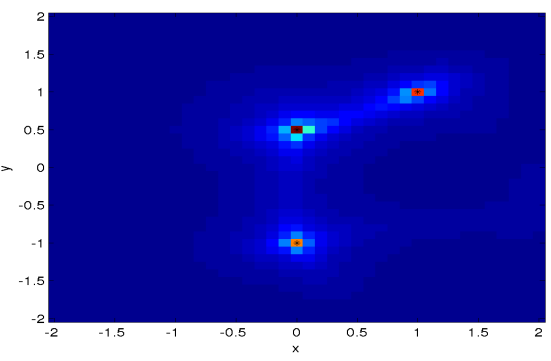

(a)

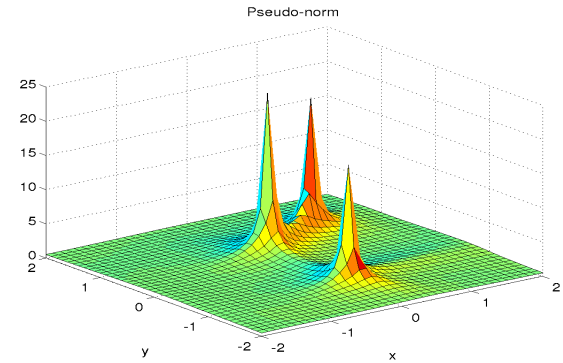

(c)

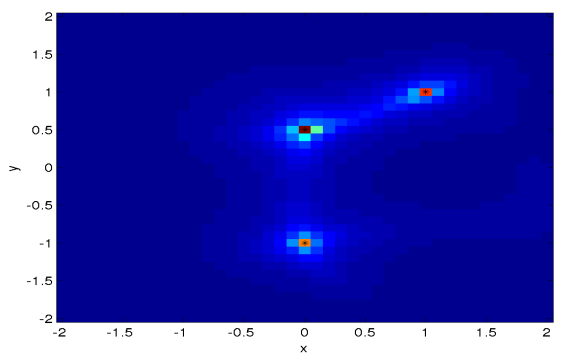

(b)

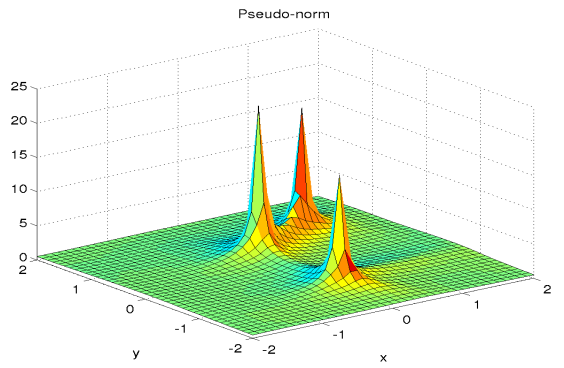

(d)

Figure 10: Born and Foldy based PP scatterings respectively from left to right with $2 \%$ random noise, $g_{i}=1$ and $\kappa=\pi$ for 3 scatterers. 
each having scattering strength 1 for the wavenumber $\kappa=1.5 \pi$ with $2 \%$ random noise in the measured far field pattern.

Figures 8 and 9 shows the results for PP and SP far field patterns in the case of Foldy model. Figure 8 shows good reconstruction for all the scatterers in both PP and SP cases even though in SP case the peaks are sharper at locations $y_{2}, y_{5}$ and $y_{13}$. Here, we can observe that the reconstruction looks better in SP case than in PP case. This is more clear in figure 9 with more number of scatterers.

Figure 10 shows the results for PP far field patterns in the case of Born approximation and Foldy model. In this case $2 \%$ random noise in the measured far field pattern is used. Here, the scatterers satisfy the diagonally dominant condition (3.61) and the reconstruction is good and similar for both the Born approximation and the Foldy model.

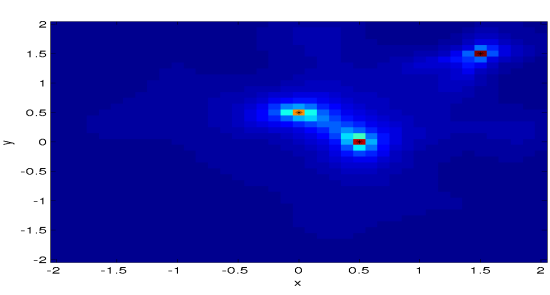

(a)

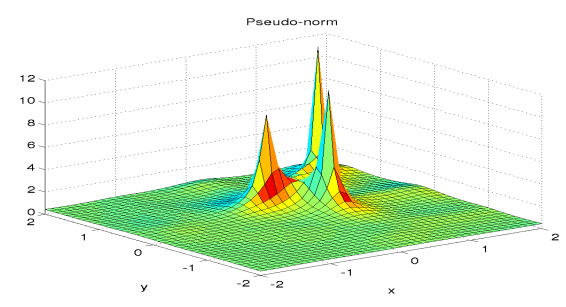

(c)

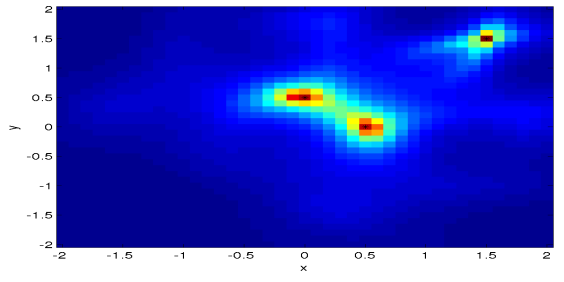

(b)

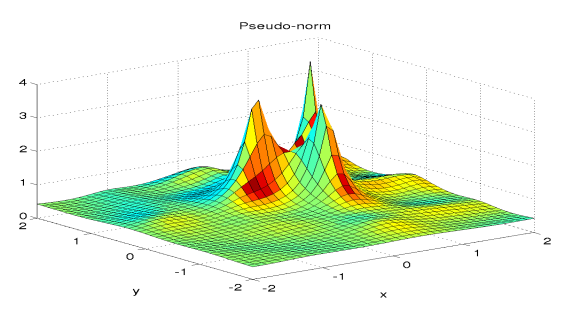

(d)

Figure 11: Born and Foldy based PP scatterings respectively from left to right with $5 \%$ random noise, $g_{i}=10$ and $\kappa=1.5 \pi$ for 3 scatterers.

Figures 11, 12, 13 and 14 are related to the three scatterers located at the points $y_{2}, y_{3}$ and $y_{14}$. Figure 11 shows the pseudo graphs for PP scattering of Born approximation and Foldy model for the wavenumber $\kappa=1.5 \pi$ with $5 \%$ random noise in the measured far field pattern. Here, scatterers violates the diagonally dominant condition (3.61) and we can see the effect of multiple scattering in the case of Foldy.

Figures 12 and 13 shows the reconstruction results for SP scattering of Born approximation and Foldy method for the wavenumber $\kappa=\pi$ with $2 \%$ and $10 \%$ random noises respectively in the measured far field pattern. In both the figures, we can see the effect of multiple scattering.

In all these figures we showed the differences between Born approximation and Foldy's method. Figure 10 is the one for which scatterers satisfies the diagonally dominant condition (3.61) and in the rest of the figures this condition is violated by the scatterers. In all the cases, we study the effect of noise level, relative distance between the obstacles comparing to their scattering strengths. It is clear that, the effect of multiple scattering is more in the reconstruction related to Foldy model (multiple scattering) than in Born approximation (weak scattering). Similarly, we see that $\mathrm{S}$ incident waves give better reconstruction than $\mathrm{P}$ incident waves since the former have shorter wavelengths than the latter, see (3.4) and remark that $\kappa_{s}>\kappa_{p}$. 


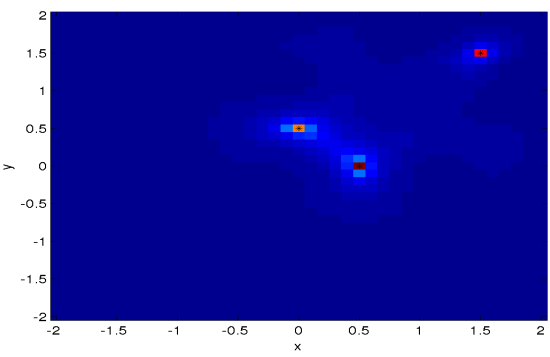

(a)

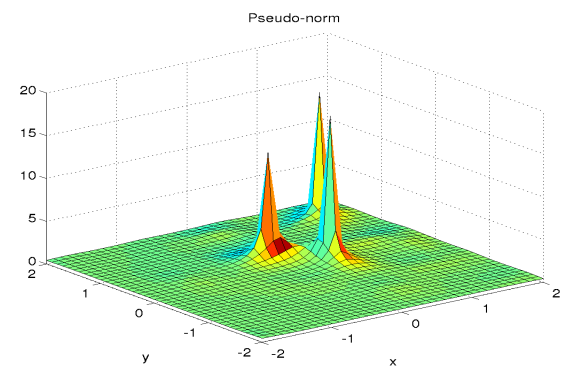

(c)

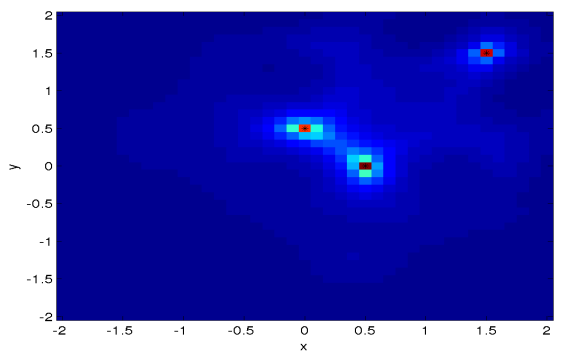

(b)

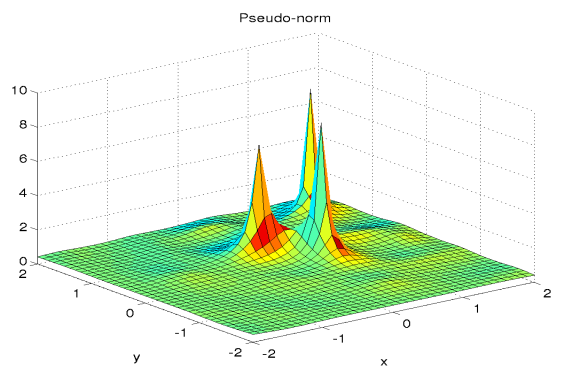

(d)

Figure 12: Born and Foldy based SP scatterings respectively from left to right with $2 \%$ random noise, $g_{i}=10$ and $\kappa=\pi$ for 3 scatterers.

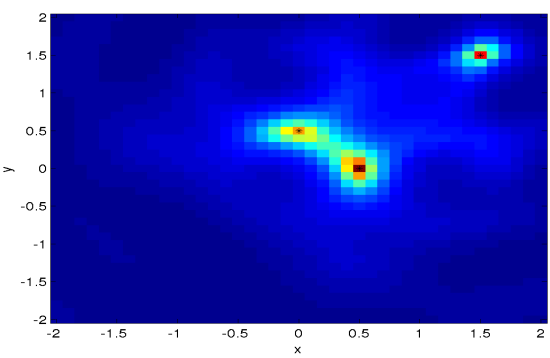

(a)

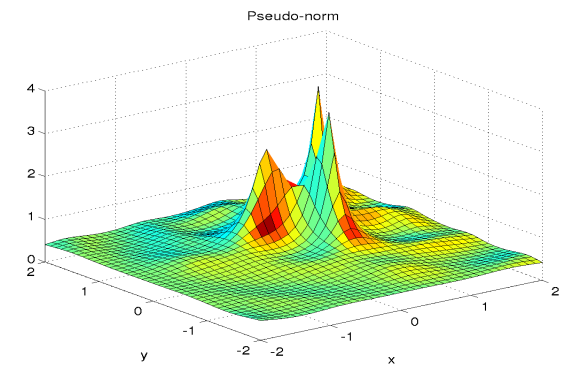

(c)

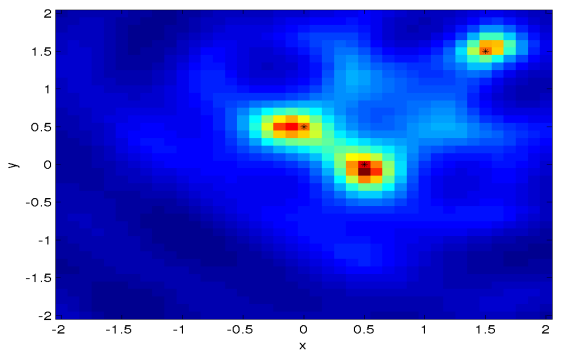

(b)

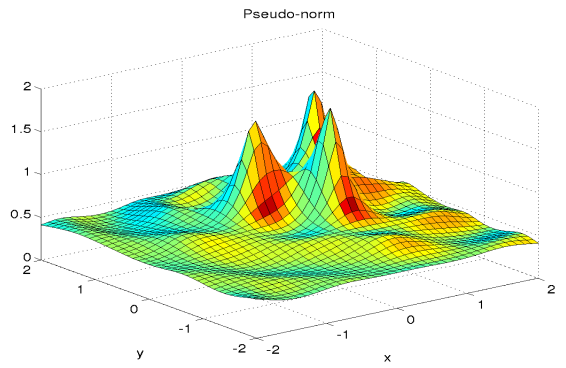

(d)

Figure 13: Weak and multiple SP scatterings respectively from left to right with $10 \%$ noise, $g_{i}=10$ and $\kappa=\pi$ for 3 scatterers. 


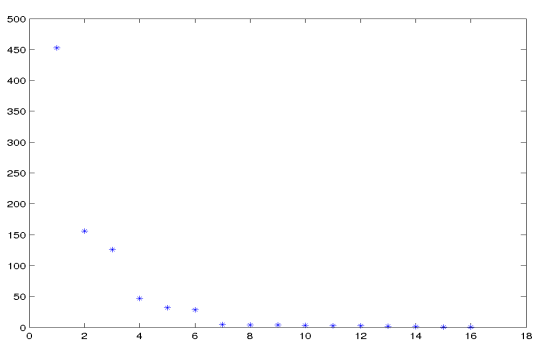

(a)

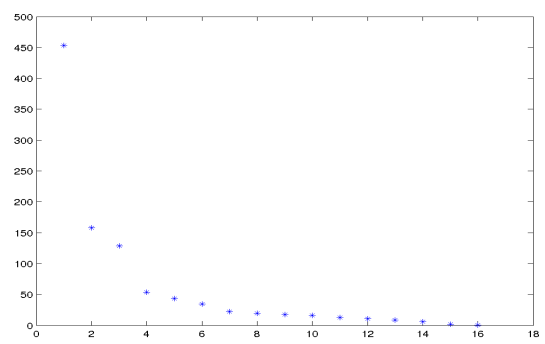

(b)

Figure 14: Singular values in the Foldy based SP scattering in presence of 3 scatterers with $2 \%$ and $5 \%$ random noises respectively from left to right $\left(g_{i}=10\right.$ and $\left.\kappa=\pi\right)$.

To finish this section, let us mention that the reconstruction depends on the choice of the signal and noise subspaces of the multi scale response matrix. For small measurement noise, these two subspaces are easy to choose due to the clear cut in the distribution of the singular values of the multi scale response matrix. However, for large noise, the distribution of the singular values are smooth and it becomes more difficult to separate the singular values of the noise and signal subspaces, for example see figure 14 for the SP case with 3 scatterers. We can observe this more clearly in elastic scattering than in acoustic scattering.

\subsection{The inverse problems for the intermediate levels of scattering}

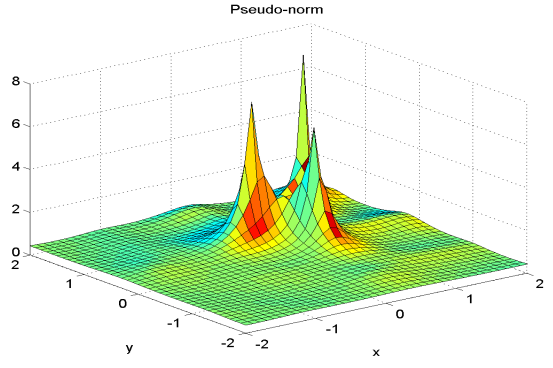

(a)

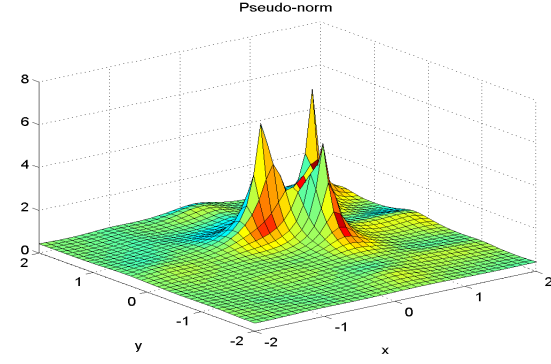

(b)

Figure 15: PP scattering for 3 scatterers with $5 \%$ noise, $g_{i}=10$ and $\kappa=1.5 \pi$. (a) 3rd level scattering (b) 8th level scattering .

We recall, see Section 3.3, that the scattered field in the $k^{\text {th }}$ level scattering is calculated by

$$
u_{k}^{s}(x)=\sum_{m=1}^{M} g_{m} G\left(x, y_{m}\right) u_{m, k}\left(y_{m}\right),
$$


with $u_{m, k}\left(y_{m}\right)$ defined recursively by

$$
u_{m, k+1}\left(y_{m}\right):=u^{i}\left(y_{m}\right)+\sum_{\substack{j=1 \\ j \neq m}}^{M} g_{j} G\left(y_{m}, y_{j}\right) u_{j, k}\left(y_{j}\right) \text { for } m=1, \ldots, M .
$$

Also recall that we can summarise the different models by the formula

$$
u_{I, k}=\sum_{l=0}^{k}(-\tilde{\mathbb{M}})^{l} u^{I} \text { for } k=0,1, \ldots
$$

where $u_{I, k}, u^{I}$ and $\tilde{\mathbb{M}}$ are as mentioned earlier in Section 3.3. Then by the similar approach as in Section 3.5.1, we obtain the factorization of the response matrix in the $k^{\text {th }}$ level scattering as

$$
F_{t}^{r}=H^{t^{*}} \tilde{T} H^{r}
$$

with $\tilde{T}=\tilde{\mathbf{g}} \sum_{l=0}^{k}(-\tilde{\mathbb{M}})^{l}, r$ (resp. $t$ ) is either $p$ or $s$ or $s^{h}$ or $s^{v}$ based on the type of the far field pattern ( resp. incident wave). Here $\tilde{\mathbf{g}}, H^{p}, H^{s}, H^{s^{h}}$ and $H^{s^{v}}$ are defined as in Section 3.5.1. To apply the MUSIC algorithm, the invertibility of the matrix $\tilde{T}$ is needed and the norm of $\tilde{\mathbb{M}}$ less than half is the sufficient condition for that in every level of scattering. In this case the reconstruction looks quite similar in all the levels of scattering when the scatterers are far enough from each other. However, we can observe the similar kind of differences which we mentioned between weak (Born) and multiple (Foldy) scatterings, as the level $k$ of the scattering increases, with respect to the condition (3.61). We can observe this in figure 15 , for the same data as in figure 11.

As discussed in Section 3.5.3, we can recover the matrix $\tilde{T} \in \mathbb{C}^{d M \times d M}$ as $\tilde{T}=I_{H^{t}} H^{t} F_{t}^{r} H^{r^{*}} I_{H^{r}}$, where $F_{t}^{r}$ is the given far field pattern in the $k^{t h}$ level scattering. By comparing this evaluated $\tilde{T}$ with its explicit form, $\tilde{\mathbf{g}} \sum_{l=0}^{k}(-\tilde{\mathbb{M}})^{l}$, in $k^{t h}$ level scattering we can recover the scattering strengths $g_{m}$ 's. In Foldy model it is clear as mentioned in Section 3.5.3 for $M$ scatterers, where as in Born model $\tilde{T}=\tilde{\mathbf{g}}$ and so the diagonal entries of $\tilde{T}$ produces the corresponding scattering strengths of the scatterers. In the case $k=1$, we have $\tilde{T}=\tilde{\mathbf{g}}-\tilde{\mathbf{g}} \tilde{\mathbb{M}}$. As we know that $\tilde{\mathbf{g}}$ is a diagonal matrix and the diagonal blocks of the size $d \times d$ of $\tilde{\mathbb{M}}$ are zero, the diagonal entries of $\tilde{T}$ are equal to the scattering strengths of the $M$ scatterers. But for intermediate level scattering $k>1$, it is difficult to recover the scattering strengths due to the complicated structure of the matrices $(-\tilde{\mathbb{M}})^{l}$, for $l=2, \ldots$, and hence of $\tilde{T}$. For this reason, as in the scalar case, we restrict ourselves to the special case of two point-like obstacles $y_{1}, y_{2}$ with the corresponding scattering strengths $g_{1}, g_{2}$. In this case using the reciprocity relation of the fundamental matrix $G(x, y)$, i.e. $G(x, y)=[G(y, x)]^{\mathbf{t}}$, we have the explicit form of $(-\tilde{\mathbb{M}})^{l}$ for $l=0,1,2, \ldots$ as follows

$$
(-\tilde{\mathbb{M}})^{l}=\left\{\begin{array}{cc}
{\left[\begin{array}{cc}
g_{1}^{\frac{l}{2}} g_{2}^{\frac{l}{2}} G^{l}\left(y_{1}, y_{2}\right) & \mathbf{0} \\
\mathbf{0} & g_{1}^{\frac{l}{2}} g_{2}^{\frac{l}{2}} G^{l}\left(y_{1}, y_{2}\right)
\end{array}\right],} & l \in 2 \mathbb{N} \cup\{0\} \\
{\left[\begin{array}{cc}
\mathbf{0} & g_{1}^{\frac{l-1}{2}} g_{2}^{\frac{l+1}{2}} G^{l}\left(y_{1}, y_{2}\right) \\
g_{1}^{\frac{l+1}{2}} g_{2}^{\frac{l-1}{2}} G^{l}\left(y_{1}, y_{2}\right) & \mathbf{0}
\end{array}\right], \quad l \in 2 \mathbb{N}-1}
\end{array}\right.
$$

Here, $\mathbf{0}$ is the zero matrix of order $d$. The matrix $(-\tilde{\mathbb{M}})^{l}$ is either diagonal or anti-diagonal by blocks of the size $d \times d$. This structure is not valid anymore for the case of more than two scatterers. From this structure, we obtain the explicit form of $\tilde{T}=\tilde{\mathbf{g}} \sum_{l=0}^{k}(-\tilde{\mathbb{M}})^{l}$ in the $k^{\text {th }}$ order scattering as follows 


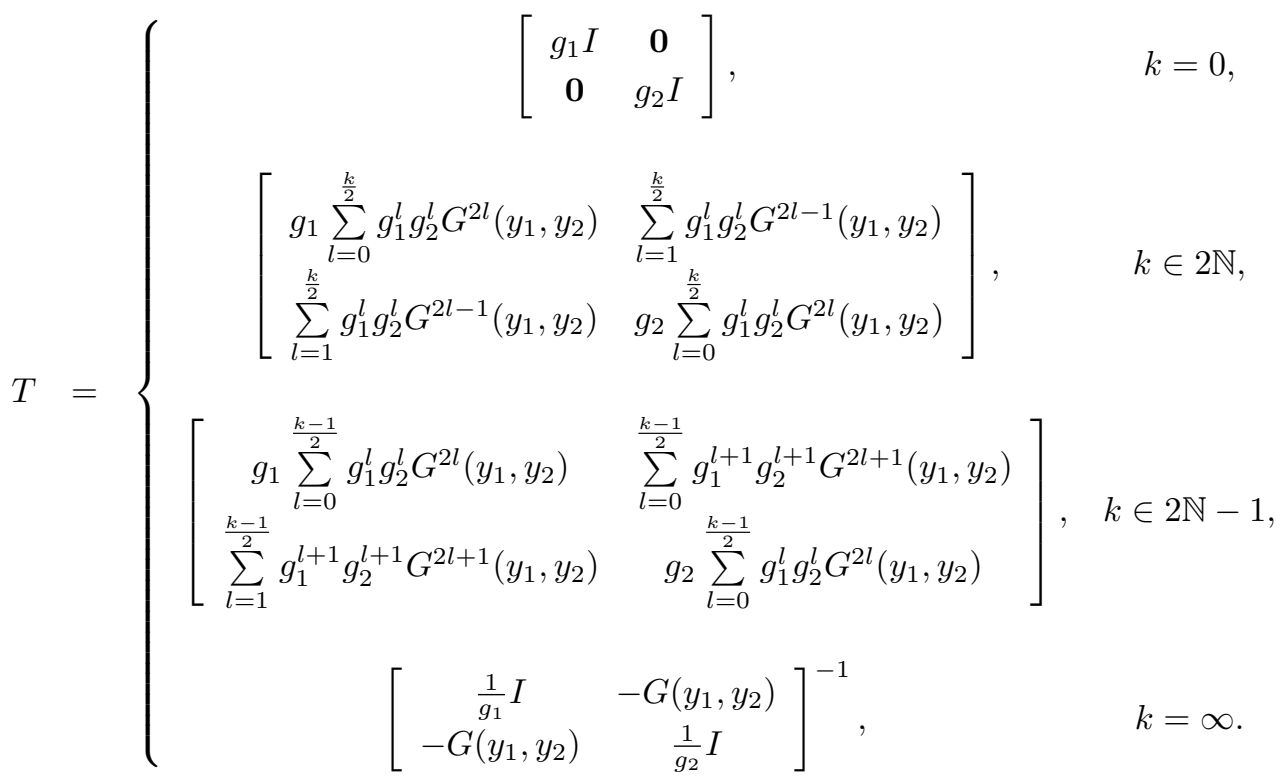

From the above explicit form of $T$, we can deduce the scattering strengths $g_{1}$ and $g_{2}$ by following the similar steps we mentioned in Section 2.4 of the acoustic scattering.

\section{Conclusion}

We used the Foldy method to model point-like scatterers and we defined the intermediate levels of scattering between Born and Foldy. Using MUSIC type algorithms, we can locate the scatterers and then recover the scattering strengths from far fields corresponding to incident plane waves. We have shown that the accuracy of reconstruction is proportional to the distance between the scatterers but inversely proportional to the wavelength, the noise in measured far field patterns, the scattering strengths appearing and to the number of point-like scatterers. In the elastic wave scattering, $\mathrm{S}$ incident waves provide more accurate reconstruction of the locations of the scatterers compared to $\mathrm{P}$ incident waves. This is true for Born, Foldy and for any intermediate level of scattering. In particular, the larger the Lamé parameter $\lambda$ the better the reconstruction with the $\mathrm{S}$ incident waves compared to the $\mathrm{P}$ incident waves. We have also proved that, one type of incident wave $(\mathrm{P}$ or $\mathrm{S})$ and one part of the far field $(\mathrm{P}$ or $\mathrm{S})$ is sufficient to locate the point-like scatterers. In addition, one part of the $\mathrm{S}$ wave (SH-wave or $\mathrm{SV}$-wave) is sufficient in the three dimensional case.

\section{Appendix: Proof of Theorem 3.1 and Theorem 3.2}

We prove the results in the 3D case, i.e. Theorem 3.2. The same proof can be applied in the 2D case as well.

\subsection{Part I - $H^{p}$ Case}

First we note that $\phi_{z, p}^{j} \in \mathcal{R}\left(H^{p *}\right), \forall j=1,2,3$ if $z \in\left\{y_{1}, \ldots, y_{M}\right\}$ because $\phi_{y_{m}, p}^{j}, m=1, \ldots, M, j=1,2,3$, are the columns of the matrix $H^{p *} \in \mathbb{C}^{N \times 3 M}$. 
We show that there exists $N_{0} \in \mathbb{N}$ such that for every point $z \in \mathbf{K} \backslash\left\{y_{1}, y_{2}, \ldots, y_{M}\right\}$, the elements of $\left\{\phi_{y_{1}, p}^{1}, \phi_{y_{1}, p}^{2}, \phi_{y_{1}, p}^{3}, \phi_{y_{2}, p}^{1}, \phi_{y_{2}, p}^{2}, \phi_{y_{2}, p}^{3}, \ldots, \phi_{y_{M}, p}^{1}, \phi_{y_{M}, p}^{2}, \phi_{y_{M}, p}^{3}, \phi_{z, p}^{j}\right\}$ are linearly independent for every $j=1,2,3$ and for all $N \geq N_{0}$. In particular, this would imply that $H^{p *}$ has maximal rank $3 \mathrm{M}$ and that $\phi_{z, p}^{j} \notin \mathcal{R}\left(H^{p^{*}}\right)$ for every $j=1,2,3$ and for all $z \in \mathbf{K} \backslash\left\{y_{1}, y_{2}, \ldots, y_{M}\right\}$. Let us deal with $j=1$ first.

Assume on the contrary that this is not the case. Then there exist sequences $N_{l} \rightarrow \infty,\left\{\lambda_{m_{t}}^{(l)}\right\} \subset \mathbb{C}^{M}$ for $t=1,2,3,\left\{z^{(l)}\right\} \subset \mathbf{K} \backslash\left\{y_{1}, y_{2}, \ldots, y_{M}\right\}$ and $\left\{\mu^{(l)}\right\} \subset \mathbb{C}$ such that

$$
\left|\mu^{l}\right|+\sum_{\substack{m=1 \\ t=1}}^{\substack{m=M \\ t=3}}\left|\lambda_{m_{t}}^{l}\right|=1
$$

and

$$
\mu^{(l)} e^{-i \kappa_{p} z^{(l)} \cdot \theta_{s}}\left(\theta_{s} \cdot e_{1}\right)+\sum_{\substack{m=1 \\ t=1}}^{\substack{m=M \\ t=3}} \lambda_{m_{t}}^{(l)} e^{-i \kappa_{p} y_{m} \cdot \theta_{s}}\left(\theta_{s} \cdot e_{t}\right)=0, \forall s=1,2, \ldots, N_{l}
$$

Since all the sequences are bounded, there exist converging subsequences $z^{(l)} \rightarrow z \in \mathbf{K},\left\{\lambda_{m_{t}}^{(l)}\right\} \rightarrow \lambda \in$ $\mathbb{C}^{3 M}$ and $\mu^{(l)} \rightarrow \mu \in \mathbb{C}$ as $l$ tends to infinity. We fix $s \in \mathbb{N}$ and let $l$ tend to infinity. Then

$$
|\mu|+\sum_{\substack{m=1 \\ t=1}}^{\substack{m=M \\ t=3}}\left|\lambda_{m_{t}}\right|=1 \text { and } \mu e^{-i \kappa_{p} z \cdot \theta_{s}}\left(\theta_{s} \cdot e_{1}\right)+\sum_{\substack{m=1 \\ t=1}}^{\substack{m=M \\ t=3}} \lambda_{m_{t}} e^{-i \kappa_{p} y_{m} \cdot \theta_{s}}\left(\theta_{s} \cdot e_{t}\right)=0 .
$$

Since it holds for every $s \in \mathbb{N}$ we conclude from the assumption on the "richness" of the set $\left\{\theta_{s}: s \in \mathbb{N}\right\}$ that

$$
\mu e^{-i \kappa_{p} z \cdot \theta}\left(\theta \cdot e_{1}\right)+\sum_{\substack{m=1 \\ t=1}}^{\substack{m=M \\ t=3}} \lambda_{m_{t}} e^{-i \kappa_{p} y_{m} \cdot \theta}\left(\theta \cdot e_{t}\right)=0, \forall \theta \in \mathbb{S}^{2} .
$$

The left hand side is the far field pattern of the function

$$
x \longmapsto \mu\left(\nabla_{z} \Phi_{\kappa_{p}}(x, z) \cdot e_{1}\right)+\sum_{\substack{m=1 \\ t=1}}^{\substack{m=M \\ t=3}} \lambda_{m_{t}}\left(\nabla_{y} \Phi_{\kappa_{p}}\left(x, y_{m}\right) \cdot e_{t}\right) .
$$

Here, $\Phi_{\kappa_{p}}$ is the fundamental solution of the Helmholtz equation with wavenumber $\kappa_{p}$. Therefore by Rellich's Lemma and unique continuation,

$$
\mu\left(\nabla_{z} \Phi_{\kappa_{p}}(x, z) \cdot e_{1}\right)+\sum_{\substack{m=1 \\ t=1}}^{\substack{m=M \\ t=3}} \lambda_{m_{t}}\left(\nabla_{y} \Phi_{\kappa_{p}}\left(x, y_{m}\right) \cdot e_{t}\right)=0, \forall x \notin\left\{z, y_{1}, y_{2}, \ldots, y_{m}\right\} .
$$

Now, we distinguish between two cases:

(A) Let $z \notin\left\{y_{1}, y_{2}, \ldots, y_{m}\right\}$. By letting $x$ tend to $z$ and then to $y_{m}$ for $m=1, \ldots, M$, we conclude that all the coefficients $\mu$ and $\lambda_{m_{t}}$ for $t=1,2,3$ and $m=1, \ldots, M$ have to vanish. Indeed,

(1) By letting $x$ tend to $z, \nabla_{z} \Phi_{\kappa_{p}}(x, z) \cdot e_{1} \longmapsto \infty$ and from (4.3), $\mu$ has to be zero. 
(2) We write

$$
\begin{aligned}
\sum_{t=1}^{3} \lambda_{m_{t}}\left(\nabla_{y} \Phi_{\kappa_{p}}\left(x, y_{m}\right) \cdot e_{t}\right)= & \frac{e^{i \kappa_{p}\left|y_{m}-x\right|}}{4 \pi\left|y_{m}-x\right|^{2}}\left(i \kappa_{p}-\frac{1}{\left|y_{m}-x\right|}\right) \\
& \left(\lambda_{m_{1}}\left(y_{m_{1}}-x_{1}\right)+\lambda_{m_{2}}\left(y_{m_{2}}-x_{2}\right)+\lambda_{m_{3}}\left(y_{m_{3}}-x_{3}\right)\right) .
\end{aligned}
$$

By taking $x=\left(y_{m_{1}}, y_{m_{2}}, x_{3}\right)$ and $x_{3}$ tending to $y_{m_{3}}$ we observe that $\lambda_{m_{3}}$ has to be zero. Similarly by considering various directions of $x$ we deduce that $\lambda_{m_{1}}, \lambda_{m_{2}}$ also vanish.

This contradicts the first equation of (4.2).

(B) Let now $z \in\left\{y_{1}, y_{2}, \ldots, y_{m}\right\}$. Without loss of generality we assume that $z=y_{1}$. By the same arguments as in part (A) we conclude that

$$
\mu+\lambda_{1_{1}}=0, \lambda_{1_{2}}=0, \lambda_{1_{3}}=0 \text { and } \lambda_{m_{t}}=0 \text { for } m=2, \ldots, M \text { and for } t=1,2,3 .
$$

Now, we write (4.1) in the following form,

$$
\begin{aligned}
& {\left[\mu^{(l)}+\lambda_{1_{1}}^{(l)}\right] e^{-i \kappa_{p} y_{1} \cdot \theta_{s}}\left(\theta_{s} \cdot e_{1}\right)+\mu^{(l)}\left[e^{-i \kappa_{p} z^{(l)} \cdot \theta_{s}}-e^{-i \kappa_{p} y_{1} \cdot \theta_{s}}\right]\left(\theta_{s} \cdot e_{1}\right) } \\
&+\sum_{p=2}^{3} \lambda_{1_{p}}^{(l)} e^{-i \kappa_{p} y_{1} \cdot \theta_{s}}\left(\theta_{s} \cdot e_{p}\right)+\sum_{\substack{m=2 \\
t=3 \\
t=1}}^{\substack{m=M \\
m_{t}}} \lambda^{(l)} e^{-i \kappa_{p} y_{m} \cdot \theta_{s}}\left(\theta_{s} \cdot e_{t}\right)=0
\end{aligned}
$$

for all $s=1,2, \ldots, N_{l}$. The quantity

$$
\rho_{l}=\left|\mu^{(l)}+\lambda_{1_{1}}^{(l)}\right|+\sum_{p=2}^{3}\left|\lambda_{1_{p}}^{(l)}\right|+\sum_{\substack{m=2 \\ t=1}}^{\substack{m=M \\ t=3}}\left|\lambda_{m_{t}}^{(l)}\right|+\left|z^{(l)}-y_{1}\right|
$$

converges to zero as $l$ tends to infinity. By Taylor's formula we have that

$$
e^{-i \kappa_{p} z^{(l)} \cdot \theta_{s}}-e^{-i \kappa_{p} y_{1} \cdot \theta_{s}}=-i \kappa_{p} \theta_{s} \cdot\left(z^{(l)}-y_{1}\right) e^{-i \kappa_{p} y_{1} \cdot \theta_{s}}+O\left(\left|z^{(l)}-y_{1}\right|^{2}\right)
$$

as $l$ tends to infinity. Replacing (4.6) in (4.5) and dividing by $\rho_{l}$ yields

$$
\begin{aligned}
\sum_{p=2}^{3} \tilde{\lambda}_{1_{p}}^{(l)} e^{-i \kappa_{p} y_{1} \cdot \theta_{s}}\left(\theta_{s} \cdot e_{p}\right)+\sum_{\substack{m=2 \\
t=1}}^{\substack{m=M \\
t=3}} \tilde{\lambda}_{m_{t}}^{(l)} e^{-i \kappa_{p} y_{m} \cdot \theta_{s}}\left(\theta_{s} \cdot e_{t}\right) & \\
+ & {\left[\tilde{\lambda}_{1_{1}}^{(l)}-i \kappa_{p} \mu^{(l)}\left(\theta_{s} \cdot a^{(l)}\right)\right] e^{-i \kappa_{p} y_{1} \cdot \theta_{s}}\left(\theta_{s} \cdot e_{1}\right)=O\left(\left|z^{(l)}-y_{1}\right|\right) }
\end{aligned}
$$

for all $s=1,2, \ldots, N_{l}$, where

$$
\tilde{\lambda}_{1_{1}}^{(l)}=\frac{\mu^{(l)}+\lambda_{1_{1}}^{(l)}}{\rho_{l}}, \tilde{\lambda}_{1_{p}}^{(l)}=\frac{\lambda_{1_{p}}^{(l)}}{\rho_{l}}, p=2,3, \tilde{\lambda}_{m_{t}}^{(l)}=\frac{\lambda_{m_{t}}^{(l)}}{\rho_{l}}, m=2,3, \ldots, M, t=1,2,3 \text { and } a^{(l)}=\frac{z^{(l)}-y_{1}}{\rho_{l}} .
$$

All these sequences are bounded, hence we can extract further subsequences $\lambda_{m_{t}}^{(l)} \rightarrow \lambda_{m_{t}}$ for $m=$ $1,2, \ldots, M, t=1,2,3$ and $a^{(l)} \rightarrow a \in \mathbb{R}^{3}$ as $l$ tends to infinity. We have that

$$
\sum_{\substack{m=1 \\ t=1}}^{\substack{m=M \\ t=3}}\left|\tilde{\lambda}_{m_{t}}^{(l)}\right|+|a|=1
$$


and

$$
\left[\tilde{\lambda}_{1_{1}}-i \kappa_{p} \mu\left(\theta_{s} \cdot a\right)\right] e^{-i \kappa_{p} y_{1} \cdot \theta_{s}}\left(\theta_{s} \cdot e_{1}\right)+\sum_{p=2}^{3} \tilde{\lambda}_{1_{p}} e^{-i \kappa_{p} y_{1} \cdot \theta_{s}}\left(\theta_{s} \cdot e_{p}\right)+\sum_{\substack{m=2 \\ t=1}}^{\substack{m=M \\ t=3}} \tilde{\lambda}_{m_{t}} e^{-i \kappa_{p} y_{m} \cdot \theta_{s}}\left(\theta_{s} \cdot e_{t}\right)=0
$$

for all $s \in \mathbb{N}$. Again, by the assumption on the set $\theta_{s}: s \in \mathbb{N}$ we conclude that this equation holds for all $\theta \in \mathbb{S}^{2}$. The left-hand side is now the far field pattern of the function

$$
\begin{aligned}
x \longmapsto & \tilde{\lambda}_{1_{1}}\left(\nabla_{y} \Phi_{\kappa_{p}}\left(x, y_{1}\right) \cdot e_{1}\right)+\mu a \cdot\left(\nabla_{y} \nabla_{y} \Phi_{\kappa_{p}}\left(x, y_{1}\right)\right) \cdot e_{1} \\
& +\sum_{p=2}^{3} \tilde{\lambda}_{1_{p}}\left(\nabla_{y} \Phi_{\kappa_{p}}\left(x, y_{1}\right) \cdot e_{p}\right)+\sum_{\substack{m=2 \\
t=3 \\
t=1}}^{\substack{m=2 \\
m_{t}}}\left(\nabla_{y} \Phi_{\kappa_{p}}\left(x, y_{m}\right) \cdot e_{t}\right) .
\end{aligned}
$$

So, by Rellich's Lemma and unique continuation again,

$$
\begin{gathered}
\tilde{\lambda}_{1_{1}}\left(\nabla_{y} \Phi_{\kappa_{p}}\left(x, y_{1}\right) \cdot e_{1}\right)+\mu a \cdot\left(\nabla_{y} \nabla_{y} \Phi_{\kappa_{p}}\left(x, y_{1}\right)\right) \cdot e_{1} \\
+\sum_{p=2}^{3} \tilde{\lambda}_{1_{p}}\left(\nabla_{y} \Phi_{\kappa_{p}}\left(x, y_{1}\right) \cdot e_{p}\right)+\sum_{\substack{m=2 \\
t=3 \\
t=1}} \tilde{\lambda}_{m_{t}}\left(\nabla_{y} \Phi_{\kappa_{p}}\left(x, y_{m}\right) \cdot e_{t}\right)=0
\end{gathered}
$$

for all $x \notin\left\{y_{1}, \ldots, y_{M}\right\}$. By letting $x$ tend to $y_{m}$ for $m=2, \ldots, M$ we conclude that all the coefficients $\tilde{\lambda}_{m_{t}}$ for $m=2, \ldots, M$ and for $t=1,2,3$ have to vanish.

By letting $x$ tend to $y_{1}$, the most singular part of the term

$$
\tilde{\lambda}_{1_{1}}\left(\nabla_{y} \Phi_{\kappa_{p}}\left(x, y_{1}\right) \cdot e_{1}\right)+\mu a \cdot\left(\nabla_{y} \nabla_{y} \Phi_{\kappa_{p}}\left(x, y_{1}\right)\right) \cdot e_{1}+\sum_{p=2}^{3} \tilde{\lambda}_{1_{p}}\left(\nabla_{y} \Phi_{\kappa_{p}}\left(x, y_{1}\right) \cdot e_{p}\right)
$$

is $\mu a \cdot\left(\nabla_{y} \nabla_{y} \Phi_{\kappa_{p}}\left(x, y_{1}\right)\right) \cdot e_{1}$ and because of (4.8), $\mu a$ should vanish. Then coming to the remaining term by taking different directions of $x$ as in Part (A) and because of (4.8), we deduce that $\tilde{\lambda}_{1_{t}}$ for $t=1,2,3$ have to vanish. From (4.2) and (4.4), we get $|\mu|=1 / 2$ and thus $a=0$.

This finally contradicts (4.7).

We have done the converse part for $j=1$. In the same manner we can show it for $j=2$ and 3 as well. These arguments prove that $H^{p^{*}}$ and so $H^{p}$ have maximal ranks.

Hence, $\phi_{z, p}^{j} \in \mathcal{R}\left(H^{p *}\right)$ iff $z \in\left\{y_{1}, \ldots, y_{M}\right\}$ for some $j=1,2,3$.

\subsection{Part II - $H^{s}$ Case}

Following the similar way as in Part I, we can prove that $\phi_{z, s}^{j} \in \mathcal{R}\left(H^{s^{*}}\right)$ iff $z \in\left\{y_{1}, \ldots, y_{M}\right\}$ for some $j=1,2,3$ by proving the maximal rank property of the matrix $H^{s *}$ and so for $H^{s}$ as well. Due to some technical differences compared to the $H^{p}$ case, we provide here the details as well.

Indeed, we show that there exists $N_{1} \in \mathbb{N}$ such that for all points all points $z \in \mathbf{K} \backslash\left\{y_{1}, y_{2}, \ldots, y_{M}\right\}$, the vectors of $\left\{\phi_{y_{1}, s}^{1}, \phi_{y_{1}, s}^{2}, \phi_{y_{1}, s}^{3}, \phi_{y_{2}, s}^{1}, \phi_{y_{2}, s}^{2}, \phi_{y_{2}, s}^{3}, \ldots, \phi_{y_{M}, s}^{1}, \phi_{y_{M}, s}^{2}, \phi_{y_{M}, z}^{3}, \phi_{z, s}^{j}\right\}$ are linearly independent for every $j$ and for all $N \geq N_{1}$. In particular, this would imply that $H^{s^{*}}$ has maximal rank $3 \mathrm{M}$ and that $\phi_{z, s}^{j} \notin \mathcal{R}\left(H^{s^{*}}\right)$ for every $j$ and for all $z \in \mathbf{K} \backslash\left\{y_{1}, y_{2}, \ldots, y_{M}\right\}$. Let us deal with $\mathrm{j}=1$ first. 
Assume on the contrary that this is not the case. Then there exist sequences $N_{l} \rightarrow \infty,\left\{\lambda_{m_{t}}^{(l)}\right\} \subset \mathbb{C}^{M}$ for $t=1,2,3,\left\{z^{(l)}\right\} \subset \mathbf{K} \backslash\left\{y_{1}, y_{2}, \ldots, y_{M}\right\}$ and $\left\{\mu^{(l)}\right\} \subset \mathbb{C}$ such that

$$
\left|\mu^{l}\right|+\sum_{\substack{m=1 \\ t=1}}^{\substack{m=M \\ t=3}}\left|\lambda_{m_{t}}^{l}\right|=1
$$

and

$$
\mu^{(l)} e^{-i \kappa_{s} z^{(l)} \cdot \theta_{s}}\left(\theta_{s}^{\perp} \cdot e_{1}\right)+\sum_{\substack{m=1 \\ t=1}}^{\substack{m=M \\ t=3}} \lambda_{m_{t}}^{(l)} e^{-i \kappa_{s} y_{m} \cdot \theta_{s}}\left(\theta_{s}^{\perp} \cdot e_{t}\right)=0, \forall s=1,2, \ldots, N_{l} .
$$

Since all of the sequences are bounded there exist converging subsequence $z^{(l)} \rightarrow z \in \mathbf{K}$ and $\left\{\lambda_{m_{t}}^{(l)}\right\} \rightarrow \lambda \in$ $\mathbb{C}^{3 M}$ and $\mu^{(l)} \rightarrow \mu \in \mathbb{C}$ as $l$ tends to infinity. We fix $s \in \mathbb{N}$ and let $l$ tend to infinity. Then

$$
|\mu|+\sum_{\substack{m=1 \\ t=1}}^{\substack{m=M \\ t=3}}\left|\lambda_{m_{t}}\right|=1 \text { and } \mu e^{-i \kappa_{s} z \cdot \theta_{s}}\left(\theta_{s}^{\perp} \cdot e_{1}\right)+\sum_{\substack{m=1 \\ t=1}}^{\substack{m=M \\ t=3}} \lambda_{m_{t}} e^{-i \kappa_{s} y_{m} \cdot \theta_{s}}\left(\theta_{s}^{\perp} \cdot e_{t}\right)=0 .
$$

Since it holds for every $s \in \mathbb{N}$ we conclude from the assumption on the "richness" of the set $\left\{\theta_{s}: s \in \mathbb{N}\right\}$ that

$$
\mu e^{-i \kappa_{s} z \cdot \theta}\left(\theta^{\perp} \cdot e_{1}\right)+\sum_{\substack{m=1 \\ t=1}}^{\substack{m=M \\ t=3}} \lambda_{m_{t}} e^{-i \kappa_{s} y_{m} \cdot \theta}\left(\theta^{\perp} \cdot e_{t}\right)=0, \forall \theta \in \mathbb{S}^{2} .
$$

We know that, $\theta^{\perp}=\alpha \theta^{\perp_{h}}+\beta \theta^{\perp_{v}}$ with $\alpha$ and $\beta$ as arbitrary constants. By taking $\alpha=1, \beta=0$, we have

$$
H^{s}=H^{s^{h}} \text { and } \theta^{\perp}=\theta^{\perp_{h}}=\frac{1}{\theta_{1}^{2}+\theta_{2}^{2}}\left(\theta_{2}^{2}+\theta_{1}^{2} \theta_{3}, \theta_{1} \theta_{2}\left(\theta_{3}-1\right),-\theta_{1}\left(\theta_{1}^{2}+\theta_{2}^{2}\right)\right) .
$$

(If we take $\alpha=0, \beta=1$, then $\theta^{\perp}=\theta^{\perp_{v}}$ and it can be used to show the maximal rank property of $H^{s}=H^{s^{v}}$.) Now, the left hand side of (4.10) is the far field pattern of the function

$$
\begin{aligned}
x \longmapsto & \mu\left(-i \kappa_{s} \frac{\partial^{2}}{\partial z_{2}^{2}} \Phi_{\kappa_{s}}(x, z)+\frac{\partial^{3}}{\partial z_{1}^{2} \partial z_{3}} \Phi_{\kappa_{s}}(x, z)\right) \\
& +\sum_{m=1}^{m=M} \lambda_{m_{1}}\left(-i \kappa_{s} \frac{\partial^{2}}{\partial y_{m_{2}^{2}}} \Phi_{\kappa_{s}}\left(x, y_{m}\right)+\frac{\partial^{3}}{\partial y_{m_{1}}^{2} \partial y_{m 3}} \Phi_{\kappa_{s}}\left(x, y_{m}\right)\right) \\
& +\sum_{m=1}^{m=M} \lambda_{m_{2}}\left(\frac{\partial^{3}}{\partial y_{m_{1}} \partial y_{m_{2}} \partial y_{m_{3}}} \Phi_{\kappa_{s}}\left(x, y_{m}\right)+i \kappa_{s} \frac{\partial^{2}}{\partial y_{m_{1}} \partial y_{m_{2}}} \Phi_{\kappa_{s}}\left(x, y_{m}\right)\right) \\
& -\sum_{m=1}^{m=M} \lambda_{m_{3}}\left(\frac{\partial^{3}}{\partial y_{m_{1}^{3}}} \Phi_{\kappa_{s}}\left(x, y_{m}\right)+\frac{\partial^{3}}{\partial y_{m_{1}} \partial y_{m_{2}^{2}}} \Phi_{\kappa_{s}}\left(x, y_{m}\right)\right) .
\end{aligned}
$$

Here, $\Phi_{\kappa_{s}}$ is the fundamental solution of the Helmholtz equation with wavenumber $\kappa_{s}$. Therefore by 
Rellich's Lemma and unique continuation,

$$
\begin{aligned}
& \mu\left(-i \kappa_{s} \frac{\partial^{2}}{\partial z_{2}^{2}} \Phi_{\kappa_{s}}(x, z)+\frac{\partial^{3}}{\partial z_{1}^{2} \partial z_{3}} \Phi_{\kappa_{s}}(x, z)\right) \\
& +\sum_{m=1}^{m=M} \lambda_{m_{1}}\left(-i \kappa_{s} \frac{\partial^{2}}{\partial y_{m_{2}}^{2}} \Phi_{\kappa_{s}}\left(x, y_{m}\right)+\frac{\partial^{3}}{\partial y_{m_{1}}^{2} \partial y_{m_{3}}} \Phi_{\kappa_{s}}\left(x, y_{m}\right)\right) \\
& +\sum_{m=1}^{m=M} \lambda_{m_{2}}\left(\frac{\partial^{3}}{\partial y_{m_{1}} \partial y_{m_{2}} \partial y_{m_{3}}} \Phi_{\kappa_{s}}\left(x, y_{m}\right)+i \kappa_{s} \frac{\partial^{2}}{\partial y_{m_{1}} \partial y_{m_{2}}} \Phi_{\kappa_{s}}\left(x, y_{m}\right)\right) \\
& -\sum_{m=1}^{m=M} \lambda_{m_{3}}\left(\frac{\partial^{3}}{\partial y_{m_{1}^{3}}} \Phi_{\kappa_{s}}\left(x, y_{m}\right)+\frac{\partial^{3}}{\partial y_{m_{1}} \partial y_{m_{2}}^{2}} \Phi_{\kappa_{s}}\left(x, y_{m}\right)\right) \\
& =0
\end{aligned}
$$

for all $x \notin\left\{z, y_{1}, y_{2}, \ldots, y_{m}\right\}$. Again, we distinguish between two cases:

(A.) Let $z \notin\left\{y_{1}, y_{2}, \ldots, y_{m}\right\}$. By letting $x$ tend to $z$ and to $y_{m}$ for $m=1, \ldots, M$ we conclude that all the coefficients $\mu$ and $\lambda_{m_{t}}$ for $t=1,2,3$ and $m=1, \ldots, M$ have to vanish. Indeed,

(1.) Taking $x=\left(z_{1}, x_{2}, z_{3}\right)$ with $x_{2}$ tending to $z_{2}$, then due to the singularity of $\frac{\partial^{2}}{\partial z_{2}^{2}} \Phi_{\kappa_{s}}(x, z)$ in (4.11), $\mu$ has to be zero.

(2.) Consider the term

$\lambda_{m_{1}}\left(-i \kappa_{s} \frac{\partial^{2}}{\partial y_{m_{2}^{2}}^{2}} \Phi_{\kappa_{s}}\left(x, y_{m}\right)+\frac{\partial^{3}}{\partial y_{m_{1}^{2}}^{2} \partial y_{m_{3}}} \Phi_{\kappa_{s}}\left(x, y_{m}\right)\right)-\lambda_{m_{3}}\left(\frac{\partial^{3}}{\partial y_{m_{1}^{3}}} \Phi_{\kappa_{s}}\left(x, y_{m}\right)+\frac{\partial^{3}}{\partial y_{m_{1}} \partial y_{m_{2}^{2}}^{2}} \Phi_{\kappa_{s}}\left(x, y_{m}\right)\right)$ $+\lambda_{m_{2}}\left(\frac{\partial^{3}}{\partial y_{m_{1}} \partial y_{m_{2}} \partial y_{m_{3}}} \Phi_{\kappa_{s}}\left(x, y_{m}\right)+i \kappa_{s} \frac{\partial^{2}}{\partial y_{m_{1}} \partial y_{m_{2}}} \Phi_{\kappa_{s}}\left(x, y_{m}\right)\right)$.

Taking $x=\left(x_{1}, y_{m_{2}}, y_{m_{3}}\right)$ and tending $x_{1}$ to $y_{m_{1}}$ we observe that $\lambda_{m_{3}}$ has to be zero due to the singularity of $\frac{\partial^{3}}{\partial y_{m_{1}^{3}}} \Phi_{\kappa_{s}}\left(x, y_{m}\right)$ in (4.11). Similarly, by considering the other directions, we can show that $\lambda_{m_{1}}, \lambda_{m_{2}}$ also vanish.

This contradicts the first equation of (4.10).

(B.) Let now $z \in\left\{y_{1}, y_{2}, \ldots, y_{m}\right\}$. Without loss of generality we also assume that $z=y_{1}$. By the same arguments as in part (A.) we conclude that

$$
\mu+\lambda_{1_{1}}=0, \lambda_{1_{2}}=0, \lambda_{1_{3}}=0 \text { and } \lambda_{m_{t}}=0 \text { for } m=2, \ldots, M \text { and for } t=1,2,3 .
$$

Now, we write (4.10) in the following form,

$$
\begin{aligned}
& {\left[\mu^{(l)}+\lambda_{1_{1}}^{(l)}\right] e^{-i \kappa_{s} y_{1} \cdot \theta_{s}}\left(\theta_{s}^{\perp} \cdot e_{1}\right)+\mu^{(l)}\left[e^{-i \kappa_{s} z^{(l)} \cdot \theta_{s}}-e^{-i \kappa_{s} y_{1} \cdot \theta_{s}}\right]\left(\theta_{s}^{\perp} \cdot e_{1}\right) } \\
&+\sum_{p=2}^{3} \lambda_{1_{p}}^{(l)} e^{-i \kappa_{s} y_{1} \cdot \theta_{s}}\left(\theta_{s}^{\perp} \cdot e_{p}\right)+\sum_{\substack{m=2 \\
t=3}}^{\lambda_{t=1}} \lambda_{m_{t}}^{(l)} e^{-i \kappa_{s} y_{m} \cdot \theta_{s}}\left(\theta_{s}^{\perp} \cdot e_{t}\right)=0
\end{aligned}
$$

for all $s=1,2, \ldots, N_{l}$. The quantity

$$
\rho_{l}=\left|\mu^{(l)}+\lambda_{1_{1}}^{(l)}\right|+\sum_{p=2}^{3}\left|\lambda_{1_{p}}^{(l)}\right|+\sum_{\substack{m=2 \\ t=1}}^{\substack{m=M \\ t=3}}\left|\lambda_{m_{t}}^{(l)}\right|+\left|z^{(l)}-y_{1}\right|
$$


converges to zero as $l$ tends to infinity. By Taylor's formula we have that

$$
e^{-i \kappa_{s} z^{(l)} \cdot \theta_{s}}-e^{-i \kappa_{s} y_{1} \cdot \theta_{s}}=-i \kappa_{s} \theta_{s} \cdot\left(z^{(l)}-y_{1}\right) e^{-i \kappa_{s} y_{1} \cdot \theta_{s}}+O\left(\left|z^{(l)}-y_{1}\right|^{2}\right)
$$

as $l$ tends to infinity. Replacing (4.14) in (4.13) and dividing by $\rho_{l}$ yields

$$
\begin{aligned}
\sum_{p=2}^{3} \tilde{\lambda}_{1_{p}}^{(l)} e^{-i \kappa_{s} y_{1} \cdot \theta_{s}}\left(\theta_{s}^{\perp} \cdot e_{p}\right)+\sum_{\substack{m=2 \\
t=1}}^{\substack{m=M \\
t=3}} \tilde{\lambda}_{m_{t}}^{(l)} e^{-i \kappa_{s} y_{m} \cdot \theta_{s}}\left(\theta_{s}^{\perp} \cdot e_{t}\right) \\
+\left[\tilde{\lambda}_{1_{1}}^{(l)}-i \kappa_{s} \mu^{(l)}\left(\theta_{s} \cdot a^{(l)}\right)\right] e^{-i \kappa_{s} y_{1} \cdot \theta_{s}}\left(\theta_{s}^{\perp} \cdot e_{1}\right)=O\left(\left|z^{(l)}-y_{1}\right|\right)
\end{aligned}
$$

for all $s=1,2, \ldots, N_{l}$, where

$$
\tilde{\lambda}_{1_{1}}^{(l)}=\frac{\mu^{(l)}+\lambda_{1_{1}}^{(l)}}{\rho_{l}}, \tilde{\lambda}_{1_{p}}^{(l)}=\frac{\lambda_{1_{p}}^{(l)}}{\rho_{l}}, p=2,3, \tilde{\lambda}_{m_{t}}^{(l)}=\frac{\lambda_{m_{t}}^{(l)}}{\rho_{l}}, m=2,3, \ldots, M, t=1,2,3 \text { and } a^{(l)}=\frac{z^{(l)}-y_{1}}{\rho_{l}} .
$$

All these sequences are bounded as well, i.e. we can extract further subsequences $\lambda_{m_{t}}^{(l)} \rightarrow \lambda_{m_{t}}$ for $m=1,2, \ldots, M, t=1,2,3$ and $a^{(l)} \rightarrow a \in \mathbb{R}^{3}$ as $l$ tends to infinity. We have that

$$
\sum_{\substack{m=1 \\ t=1}}^{\substack{m=M \\ t=3}}\left|\tilde{\lambda}_{m_{t}}^{(l)}\right|+|a|=1
$$

and

$$
\left[\tilde{\lambda}_{1_{1}}-i \kappa_{s} \mu\left(\theta_{s} \cdot a\right)\right] e^{-i \kappa_{s} y_{1} \cdot \theta_{s}}\left(\theta_{s}^{\perp} \cdot e_{1}\right)+\sum_{p=2}^{3} \tilde{\lambda}_{1_{p}} e^{-i \kappa_{s} y_{1} \cdot \theta_{s}}\left(\theta_{s}^{\perp} \cdot e_{p}\right)+\sum_{\substack{m=2 \\ t=1}}^{\substack{m=M \\ t=3}} \tilde{\lambda}_{m_{t}} e^{-i \kappa_{s} y_{m} \cdot \theta_{s}}\left(\theta_{s}^{\perp} \cdot e_{t}\right)=0
$$

for all $s \in \mathbb{N}$. Again, by the assumption on the set $\theta_{s}: s \in \mathbb{N}$ we conclude that this equation holds for all $\theta \in \mathbb{S}^{2}$. The left-hand side is now the far field pattern of the function

$$
\begin{aligned}
x \longmapsto & \mu a \cdot \nabla_{y_{1}}\left(-i \kappa_{s} \frac{\partial^{2}}{\partial y_{1}^{2}} \Phi_{\kappa_{s}}\left(x, y_{1}\right)+\frac{\partial^{3}}{\partial y_{1}^{2} \partial y_{13}} \Phi_{\kappa_{s}}\left(x, y_{1}\right)\right) \\
& +\sum_{m=1}^{m=M} \tilde{\lambda}_{m_{1}}\left(-i \kappa_{s} \frac{\partial^{2}}{\partial y_{m_{2}}^{2}} \Phi_{\kappa_{s}}\left(x, y_{m}\right)+\frac{\partial^{3}}{\partial y_{m 1}^{2} \partial y_{m 3}} \Phi_{\kappa_{s}}\left(x, y_{m}\right)\right) \\
& +\sum_{m=1}^{m=M} \tilde{\lambda}_{m_{2}}\left(\frac{\partial^{3}}{\partial y_{m_{1}} \partial y_{m_{2}} \partial y_{m_{3}}} \Phi_{\kappa_{s}}\left(x, y_{m}\right)+i \kappa_{s} \frac{\partial^{2}}{\partial y_{m_{1}} \partial y_{m_{2}}} \Phi_{\kappa_{s}}\left(x, y_{m}\right)\right) \\
& -\sum_{m=1}^{m=M} \tilde{\lambda}_{m_{3}}\left(\frac{\partial^{3}}{\partial y_{m_{1}^{3}}} \Phi_{\kappa_{s}}\left(x, y_{m}\right)+\frac{\partial^{3}}{\partial y_{m_{1}} \partial y_{m_{2}}^{2}} \Phi_{\kappa_{s}}\left(x, y_{m}\right)\right) .
\end{aligned}
$$


So, by Rellich's Lemma and unique continuation again,

$$
\begin{aligned}
& \mu a \cdot \nabla_{y_{1}}\left(-i \kappa_{s} \frac{\partial^{2}}{\partial y_{12}^{2}} \Phi_{\kappa_{s}}\left(x, y_{1}\right)+\frac{\partial^{3}}{\partial y_{1}^{2} \partial y_{13}} \Phi_{\kappa_{s}}\left(x, y_{1}\right)\right) \\
& +\sum_{m=1}^{m=M} \tilde{\lambda}_{m_{1}}\left(-i \kappa_{s} \frac{\partial^{2}}{\partial y_{m_{2}}} \Phi_{\kappa_{s}}\left(x, y_{m}\right)+\frac{\partial^{3}}{\partial y_{m_{1}}^{2} \partial y_{m_{3}}} \Phi_{\kappa_{s}}\left(x, y_{m}\right)\right) \\
& +\sum_{m=1}^{m=M} \tilde{\lambda}_{m_{2}}\left(\frac{\partial^{3}}{\partial y_{m_{1}} \partial y_{m_{2}} \partial y_{m_{3}}} \Phi_{\kappa_{s}}\left(x, y_{m}\right)+i \kappa_{s} \frac{\partial^{2}}{\partial y_{m_{1}} \partial y_{m_{2}}} \Phi_{\kappa_{s}}\left(x, y_{m}\right)\right) \\
& -\sum_{m=1}^{m=M} \tilde{\lambda}_{m_{3}}\left(\frac{\partial^{3}}{\partial y_{m_{1}^{3}}} \Phi_{\kappa_{s}}\left(x, y_{m}\right)+\frac{\partial^{3}}{\partial y_{m_{1}} \partial y_{m_{2}}^{2}} \Phi_{\kappa_{s}}\left(x, y_{m}\right)\right) \\
& =0
\end{aligned}
$$

for all $x \notin\left\{y_{1}, \ldots, y_{M}\right\}$. By letting $x$ tend to $y_{m}$ for $m=2, \ldots, M$, we conclude that all the coefficients $\tilde{\lambda}_{m_{t}}$ for $m=2, \ldots, M$ and for $t=1,2,3$ have to vanish.

By letting $x$ tend to $y_{1}$, the most singular part of the term

$$
\begin{aligned}
& \mu a \cdot \nabla_{y_{1}}\left(-i \kappa_{s} \frac{\partial^{2}}{\partial y_{12}^{2}} \Phi_{\kappa_{s}}\left(x, y_{1}\right)+\frac{\partial^{3}}{\partial y_{11}^{2} \partial y_{13}} \Phi_{\kappa_{s}}\left(x, y_{1}\right)\right)+\tilde{\lambda}_{1_{1}}\left(-i \kappa_{s} \frac{\partial^{2}}{\partial y_{12}^{2}} \Phi_{\kappa_{s}}\left(x, y_{1}\right)+\frac{\partial^{3}}{\partial y_{1}^{2} \partial y_{13}} \Phi_{\kappa_{s}}\left(x, y_{1}\right)\right) \\
& +\tilde{\lambda}_{1_{2}}\left(\frac{\partial^{3}}{\partial y_{11} \partial y_{12} \partial y_{13}} \Phi_{\kappa_{s}}\left(x, y_{1}\right)+i \kappa_{s} \frac{\partial^{2}}{\partial y_{11} \partial y_{12}} \Phi_{\kappa_{s}}\left(x, y_{1}\right)\right)-\tilde{\lambda}_{1_{3}}\left(\frac{\partial^{3}}{\partial y_{11}^{3}} \Phi_{\kappa_{s}}\left(x, y_{1}\right)+\frac{\partial^{3}}{\partial y_{11} \partial y_{12}^{2}} \Phi_{\kappa_{s}}\left(x, y_{1}\right)\right)
\end{aligned}
$$

is $\mu a \cdot \nabla_{y_{1}}\left(-i \kappa_{s} \frac{\partial^{2}}{\partial y_{12}^{2}} \Phi_{\kappa_{s}}\left(x, y_{1}\right)+\frac{\partial^{3}}{\partial y_{1}^{2} \partial y_{13}} \Phi_{\kappa_{s}}\left(x, y_{1}\right)\right)$ and because of (4.16), $\mu a$ should vanish. Then coming to the remaining term by taking different directions of $x$ as in Part (A) and because of (4.16), we deduce that $\tilde{\lambda}_{1_{t}}$ for $t=1,2,3$ have to vanish. Now, from (4.10) and (4.12), we get $|\mu|=1 / 2$ and thus $a=0$.

This finally contradicts (4.15).

We have done the converse part for $j=1$. In the same manner we can show it for $j=2$ and 3 as well. From these arguments we get the maximal rank property of the matrix $H^{s^{*}}$ and so for $H^{s}$.

\section{References}

[1] C. J. S. Alves and R. Kress. On the far-field operator in elastic obstacle scattering. IMA J. Appl. Math., 67(1):1-21, 2002.

[2] S. Albeverio, F. Gesztesy, R. Høegh-Krohn and H. Holden. Solvable models in quantum mechanics. AMS Chelsea Publishing, Providence, RI, second edition, 2005.

[3] H. Ammari. An Introduction to Mathematics of Emerging Biomedical Imaging. Springer-Verlag, Berlin, 2008.

[4] H. Ammari, P. Calmon, and E. Iakovleva. Direct elastic imaging of a small inclusion. SIAM J. Imaging Sci., 1(2):169-187, 2008.

[5] H. Ammari, P. Garapon, H. Kang, and H. Lee. A method of biological tissues elasticity reconstruction using magnetic resonance elastography measurements. Quart. Appl. Math., 66(1):139-175, 2008. 
[6] H. Ammari, H. Kang, G. Nakamura, and K. Tanuma. Complete asymptotic expansions of solutions of the system of elastostatics in the presence of an inclusion of small diameter and detection of an inclusion. J. Elasticity, 67(2):97-129, 2002.

[7] D. Colton and R. Kress. Inverse acoustic and electromagnetic scattering theory. Springer-Verlag, Berlin, second edition, 1998.

[8] D. L. Colton and R. Kress. Integral equation methods in scattering theory. Pure and Applied Mathematics (New York). John Wiley \& Sons Inc., New York, 1983. A Wiley-Interscience Publication.

[9] G. Dassios, K. Kiriaki and D. Polyzos. On the scattering amplitudes for elastic waves. Z. Angew. Math. Phys., 38(6):856-873, 1987.

[10] G. Dassios and R. Kleinman. Low frequency scattering. Oxford Mathematical Monographs. The Clarendon Press Oxford University Press, New York, 2000. Oxford Science Publications.

[11] A. J. Devaney, E. A. Marengo, and F. K. Gruber. Time-reversal-based imaging and inverse scattering of multiply scattering point targets. J. Acoust. Soc. Am, 118:3129-3138, Nov. 2005.

[12] P. de Vries, D. V. van Coevorden, and A. Lagendijk. Point scatterers for classical waves. Rev. Mod. Phys., 70:447-466, Apr 1998.

[13] R. Fazli and M. Nakhkash. An analytical approach to estimate the number of small scatterers in 2d inverse scattering problems. Inverse Problems, 28(7):075012, 2012.

[14] L. L. Foldy. The multiple scattering of waves. I. General theory of isotropic scattering by randomly distributed scatterers. Phys. Rev. (2), 67:107-119, 1945.

[15] F. Gesztesy and A. G. Ramm. An inverse problem for point inhomogeneities. Methods Funct. Anal. Topology, 6(2):1-12, 2000.

[16] D. Gintides and M. Sini. Identification of obstacles using only the scattered P-waves or the scattered S-waves. IPI, 6(1):39-55, 2012.

[17] D. Gintides, M. Sini and N. T. Thành. Detection of point-like scatterers using one type of scattered elastic waves. J. Comput. Appl. Math., 236(8):2137-2145, 2012.

[18] G. Hu and M. Sini. Elastic scattering by finitely many point-like obstacles. Preprint available at: http://www.ricam.oeaw.ac.at/people/page/sini/publications.html

[19] H. Kang, E. Kim, and J.-Y. Lee. Identification of elastic inclusions and elastic moment tensors by boundary measurements. Inverse Problems, 19(3):703-724, 2003.

[20] A. Kirsch and N. Grinberg. The factorization method for inverse problems, volume 36 of Oxford Lecture Series in Mathematics and its Applications. Oxford University Press, Oxford, 2008.

[21] V. D. Kupradze. Potential methods in the theory of elasticity. Israel Program for Scientific Translations, Jerusalem, 1965. 
[22] V. D. Kupradze, T. G. Gegelia, M. O. Basheleǔshvili and T. V. Burchuladze. Three-dimensional problems of the mathematical theory of elasticity and thermoelasticity. North-Holland Publishing Co., Amsterdam, 1979.

[23] E. A. Marengo and F. K. Gruber. Subspace-based localization and inverse scattering of multiply scattering point targets. EURASIP J. Appl. Signal Process., 2007(1):192-192, Jan. 2007.

[24] E. A. Marengo, F. K. Gruber, and M. Jasa. Non-iterative analytical formula for inverse scattering of multiply scattering point targets. J. Acoust. Soc. Am, 120:3782-3788, 2006.

[25] P. A. Martin. Multiple scattering, volume 107 of Encyclopedia of Mathematics and its Applications. Cambridge University Press, Cambridge, 2006. 